\title{
Astrocytic GABA Transporter controls sleep by modulating GABAergic signaling in Drosophila circadian neurons
}

\author{
Ratna Chaturvedi ${ }^{1}$, Tobias Stork ${ }^{1,2}$, ChunYan Yuan ${ }^{1}$, Marc R. Freeman ${ }^{1,2}$, Patrick \\ Emery $^{1, *}$. \\ ${ }^{1}$ Department of Neurobiology, University of Massachusetts Medical School, Worcester, \\ MA 01605, USA. \\ ${ }^{2}$ Vollum Institute, Oregon Health \& Science University, Portland, OR 97239, USA. \\ * Corresponding author. Email: Patrick.emery@umassmed,edu, Tel: +1-508-856-6599
}

\section{Highlights:}

- A hypomorphic GABA transporter (gat) mutation increases Drosophila sleep.

- Astrocytic GAT controls sleep amount and consolidation.

- GAT maintains GABA tone on wake-promoting l-LNvs circadian neurons.

- GAT functions upstream of $\mathrm{GABA}_{\mathrm{A}}$ receptor RDL and its modulators WAKE and DNlg4 to modulate sleep.

\section{Summary:}

A precise balance between sleep and wakefulness is essential to sustain a good quality of life and optimal brain function. GABA is known to play a key and conserved role in sleep control, and GABAergic tone should therefore be tightly controlled in sleep circuits. Here we examined the role of the astrocytic GABA transporter (GAT) in sleep regulation using Drosophila melanogaster. We found that a hypomorphic gat mutation ( gat $^{33-1}$ ) increased sleep amount, decreased sleep latency, and increased sleep consolidation. Interestingly, sleep defects were suppressed when $g t^{33-1}$ was combined with a mutation disrupting wide-awake (wake), a gene that regulates the cell-surface levels of the GABA receptor Resistance to Dieldrin (RDL) in the wake-promoting large ventral lateral neurons (l-LNvs). Moreover, RNAi knockdown of $r d l$ and its modulator $d n l g 4$ in these circadian neurons also suppressed $g^{3} t^{33-1}$ sleep phenotypes. Brain immunohistochemistry showed that GAT-expressing astrocytes were located near RDL-positive 1-LNvs cell bodies and dendritic processes. We conclude that astrocytic GAT decreases GABAergic tone and RDL activation in arousal promoting LNvs, thus determining proper sleep amount and quality in Drosophila

Keywords: Sleep, Astrocytes, GAT, GABA, large ventral lateral clock neurons (1-LNvs). 


\section{Introduction:}

Sleep is evolutionary conserved in invertebrates and vertebrates ${ }^{1,2,3}$. The need for sleep is fundamental to the optimal function of the brain and the body, and thus for health ${ }^{4,5}$. To date, most of the sleep regulatory pathways, molecules, and neurotransmitters that have been identified are active in neurons ${ }^{6,7}$. However, evidence is growing for an important role of glia, although this role remains poorly defined ${ }^{8,9,10,11}$.

There are two key components that regulate sleep behavior in humans and other animals: circadian control and sleep homeostasis ${ }^{12,13}$. Their precise coordination determines the timing and amount of sleep. One of the important mechanisms that regulates sleep and wake cycle involves fine-tuning between firing of wake promoting and sleep promoting neurons ${ }^{14,15}$. Gamma-aminobutyric acid (GABA) primarily serves as an inhibitory neurotransmitter in adults and plays a key role in fine-tuning neuronal firing by inhibiting or dampening excitatory signal ${ }^{16,17}$. Its activity is mediated by GABA receptors present either post-synaptic to GABAergic neurons or nearby extra-synaptic locations ${ }^{18,19,20}$. Timing of sleep is determined by circadian neurons ${ }^{12,13,21}$, whose activity is fine-tuned by GABA signaling in mammals as well as in Drosophila ${ }^{15,22,23,24}$. In mammals, many SCN neurons are GABAergic and require GABA signaling to control robust circadian behavior of sleep-wake cycle ${ }^{25}$. In Drosophila, the circadian large ventral lateral neurons (1-LNvs) facilitate light-dependent arousal by releasing Pigment-Dispersing Factor (PDF), a neuropeptide signaling molecule ${ }^{26,27}$. The I-LNvs receive GABA inhibitory inputs through the $\mathrm{GABA}_{\mathrm{A}}$-receptor RDL (Resistance to Dieldrin) and $\mathrm{GABA}_{\mathrm{B}}$ receptors, which thus promote sleep ${ }^{24,28,29}$. Blocking GABA inputs in I-LNvs increases wakefulness ${ }^{24,30}$. Interestingly, the circadian clock generates daily rhythms in RDL levels and clustering at the cell surface, which precisely controls the timing of activation and inhibition of the 1-LNvs ${ }^{31,32,33}$.

Regulation of GABAergic tone at synapses also depends on astrocytes to clear GABA after its release into the extracellular space ${ }^{34,35}$. In Drosophila, astrocytes express a single GABA transporter (GAT). GAT was previously shown to be expressed in CNS astrocytes but not in neurons ${ }^{36}$. GAT depletion produces defects in larval crawling and suppresses genetically induced seizures in adult flies ${ }^{36,37}$. Interestingly, there is a link between epileptic seizures and sleep ${ }^{38,39}$. Some patients suffering from epilepsy only have seizures during sleep. Furthermore, poor quality sleep can increase the frequency of seizures in epileptic patients ${ }^{40,41}$. Here, using Drosophila melanogaster, we identified a critical role for GAT in the control of sleep. We found that astrocytic GAT controls sleep by modulating GABAergic signaling in wake-promoting LNvs. 


\section{Results:}

\section{A hypomorphic GABA transporter $\left(\right.$ gat $\left.^{33-1}\right)$ mutation increases sleep}

In the course of generating null mutants for the gaba transporter (gat) gene using a transcription activator-like effector nuclease (TALEN)-based approach ${ }^{37}$, we were also able to isolate a hypomorphic allele $\left(\right.$ gat $\left.^{33-1}\right)$. gat null mutants are embryonic lethal ${ }^{37}$. While hypomorphic $g a t^{33-1}$ mutants were also lethal over the gat deficiency $D f(4) g a t^{464 k b}$, they were homozygous viable. The $g t^{33-1}$ allele we obtained harbored a 7-nucleotide deletion in the first coding exon, that would result in a very early frameshift (Figures 1A and 1B). Since such an early termination would be expected to result in an amorphic, embryonic lethal mutation we tested if protein was still being made in the hypomorphic gat mutant using anti-GAT antibodies directed against $\mathrm{N}$ - and $\mathrm{C}$-terminal peptides ${ }^{37}$. Indeed, staining of gat $^{33-1}$ mutant larval brains revealed that GAT protein could still be detected in astrocytes, albeit at very reduced levels, using the C-terminal anti-GAT antibody, while GAT specific signal in astrocytes using the $\mathrm{N}$-terminal antibody was abolished (Figures S1A-S1D). This data suggests that in gat $^{33-1}$ mutants, a downstream methionine is utilized as an alternative start codon leading to a truncation of the cytoplasmic N-terminus of the GAT protein. Two downstream methionines preceding the first transmembrane domain could reasonably be expected to lead to hypomorphic Nterminal truncations. Their use would result in N-terminal deletions of 22 or 64 amino acids, respectively (Figures 1C and 1D). We thus conducted Western blots using the Nand C-terminal anti-GAT antisera to compare protein levels and gel mobility in head extracts of wild type and $g a t^{33-1}$ mutants. Strikingly, in gat $^{33-1}$ extracts, a weak band migrating slightly faster than wild-type GAT was detectable with the C-terminal antibody, but not with the $\mathrm{N}$-terminal antiserum. We therefore conclude that gat $^{33-1}$ is indeed a hypomorphic mutation producing low levels of truncated GAT (Figure 1E).

To test whether GAT expression is under circadian control, $w^{1118}$ flies were collected every $4 \mathrm{~h}$ for $24 \mathrm{~h}$ after 3 days of entrainment to a 12:12 h light: dark (LD) cycle. No significant time-dependent change was observed in GAT level, while the circadian pacemaker protein PERIOD (PER), showed its typical abundance rhythm (Figures 1F; Figure S1E). We then monitored circadian locomotor rhythms of gat $^{33-1}$ mutant flies. Under a standard $12: 12 \mathrm{~h}$ light: dark (LD) cycle, we observed the expected morning and evening increases in locomotor activity anticipating the light-on and -off transitions (Figure S1F). In constant darkness (DD), the period of circadian behavior of mutant flies was very slightly but reproducibly shorter than controls (Figure 1G). We also observed a significant reduction in the percentage of rhythmic flies (Figure 1H). Increase in GABAergic tone might thus have a mild impact on the functioning of the circadian neural network. Strikingly, gat $^{33-1}$ flies showed a clear reduction in locomotor activity compared to control $\left(w^{1118}\right)$ flies, indicating that $g a t^{33-1}$ flies are either less active while awake or sleeping more (Figure S1F and G). To distinguish between these two possibilities, we 
monitored sleep and activity while awake in control and $g a t^{33-1}$ flies. We found that $g a t^{33-}$ ${ }^{l}$ males slept more than controls during the day and the night (Figures $2 \mathrm{~A}$ and B). We also examined the quality of sleep by measuring mean sleep episode lengths and episode numbers. gat $^{33-1}$ males showed significantly extended sleep episode length while the number of episodes (sleep frequency) was reduced (Figures 2C and 2D). This indicates that gat $^{33-1}$ sleep was more consolidated compared to wild type control. Logically, mean wake episode lengths were reduced in $g a t^{33-1}$ males (Figure 2E). We also found that the day and night sleep onset latencies of gat $^{33-1}$ were strikingly reduced compared to wildtype (Figure 2F). gat $^{33-1}$ males also slept more in DD (Figures $2 \mathbf{G}$ and $2 \mathbf{H}$ ).

In the standard sleep assay we used, a sleep episode is defined as a minimum of 5 minutes of inactivity. It is thus important to determine whether locomotion defects or general hypoactivity might be responsible for what appears to be a sleep defect. We therefore measured the mean activity counts of flies per minute while moving (i.e., while awake). The activity of male gat $^{33-1}$ was very slightly reduced (Figure 2J), but this marginal decrease in activity while awake would be unlikely to explain the strong sleep phenotypes. Moreover, such reduction was not observed in DD even though mutant males slept more (Figure 2I), and subtle changes in activity while awake did not correlate with sleep phenotypes in subsequent experiments. We therefore conclude that $g a t^{33-1}$ males indeed sleep more than wild-type flies but are otherwise normally active.

Sleep is sexually dimorphic ${ }^{42,43,44}$. Hence, we measured sleep in mated gat $^{33-1}$ females as well (Figures S2A-S2G). Female gat $^{33-1}$ flies exhibited excess sleep with increased consolidation during night and reduced latency, as observed in male gat $^{33-1}$ flies. $^{-}$ Surprisingly, contrary to males the duration of sleep episodes during daytime was reduced, and the number of sleep episodes were increased (Figures S2C and S2D). However, for unknown reasons, daytime sleep consolidation parameters (sleep episode length and numbers) proved to be inconsistent across experiments in both males and females and will thus not be discussed further. On the other hand, nighttime sleep consolidation was consistent across experiments.

Collectively, our results indicate that GAT modulates sleep. Decreased GAT activity increases overall sleep amount and night-rime sleep consolidation.

\section{GAT functions in astrocytes to regulate sleep}

GAT has been shown previously to act predominantly in astrocytes ${ }^{36,37}$. We thus expressed wild-type GAT in astrocytes of $\mathrm{gat}^{33-1}$ mutant males using the alrm-Gal4 driver 35. As expected, astrocyte-specific expression of wild-type GAT in a mutant background reduced sleep amounts in both male and female mutant flies (Figures 3A and 3B; Figure S3A-S3F). Daytime sleep amount was rescued in males, but night-time sleep amount was 
not perfectly rescued (see discussion). Night-time sleep consolidation and daytime sleep latency were corrected with astrocytic GAT expression in both males and females gat $^{331}$ mutants (Figures 3C, 3D and 3E; Figures S3C, S3D and S3E). Night-time sleep latency was however not corrected. Of note, the UAS-gat transgene weakly corrected gat $^{33-1}$ mutant phenotypes on its own, probably because of leaky expression since this transgene contains intronic sequences ${ }^{37}$. Mean activity per minutes while moving did not differ significantly amongst the rescued flies and mutant controls (Figure 3F; Figure S3F), demonstrating as mentioned above that the gat $^{33-1}$ sleep phenotypes are not correlated and thus not caused by a subtle change in overall activity (Figure 2F).

The failure of rescuing night-time sleep latency with astrocytic GAT expression was unexpected. We therefore used a previously described genomic rescue construct (1b12) 37. As expected, this construct corrected most sleep parameters in gat $^{33-1}$ mutant flies (Figures 3A-3F; Figure S3A-S3F), further supporting that the gat $^{33-1}$ mutation caused most sleep phenotypes. Actually, we even observed a slight over-correction of several sleep parameters (Figure 3; Figure S3). Nevertheless, night-time sleep latency was not rescued. We therefore cannot ascertain thar the late-night sleep latency phenotype is caused by loss of GAT, although results described below would support this idea (see also discussion).

\section{GAT-expressing astrocytes are present near RDL-positive l-LNvs}

GABAergic neurons are numerous in the fly brain, but it is well known that a subset of them regulate the activity of the arousal-promoting 1-LNvs, a well-characterized group of circadian neurons, through activation of the $\mathrm{GABA}_{\mathrm{A}}$ receptor RDL. GAT might thus regulate sleep by controlling GABA's impact on the 1-LNvs. We would thus predict that GAT-positive astrocytes would be located close to 1-LN projections. We therefore stained brains with different markers. To locate the 1-LNvs, we used PDF antibodies. PDF is a neuropeptide that is present in cell bodies and projections of both small and large LNvs 45 . The l-LNvs are easily recognizable by their projection patterns in the medulla of the optic lobe and the large size of their cell body. To locate astrocyte processes, we expressed membrane-bound GFP with alrm-Gal4. Fly brains were thus immunostained with anti-GFP, RDL and PDF. As expected, the soma and projections of I-LNvs were positives for both PDF and RDL, (Figure 4). We also found that the astrocytes extended their processes towards both the soma and dendrites of I-LNvs (Figures 4A, a-a3 and video-S1, Figures 4A, b-b3 and video-S2). Both soma and dendrites have been found to express $\mathrm{RDL}^{28}$, and indeed RDL puncta were located in the vicinity of astrocytes (Figure 4A, c). The Z-stack orthogonal cross-projections (Figures 4B, a-d, d' and d") further confirmed the close physical presence of astrocytes near RDL positive LNvs. The Z-stack projections with orthogonal crosses also revealed that astrocytes expanded their processes towards dendritic projections of I-LNvs in the medulla (Figures 4C, a-d, d', and d"). 
Next, we examined whether astrocytic GAT could be found close to PDF neurons (Figures 4D, 4E and 4F). Astrocytes close to I-LNvs marked by PDF positive staining showed strong GAT expression (Figures 4D, a-a3 and video-S3). Furthermore, we observed that astrocytic GATs are located in close proximity to PDF-positive neuronal projections in the medulla (Figures 4D, b-b3 and video-S4). Z-stack projections with orthogonal cross show that the astrocytic processes near the I-LNvs in the central brain (Figures 4E, a-d, d' and d") as well as in the medulla (Figures 4F, a-d, d', and d") were strongly expressing GAT.

\section{Loss of wide-awake (wake) suppresses the gat $^{33-1}$ sleep phenotypes}

WIDE-AWAKE (WAKE) works downstream of the circadian clock to control sleep. It interacts with the $\mathrm{GABA}_{\mathrm{A}}$ receptor RDL and increases RDL expression and localization to the cell surface of wake-promoting I-LNvs ${ }^{31}$. As a result, the excitability of I-LNvs changes during the course of the day. This mechanism is important for the transition from wakefulness to sleep, and wake mutant flies show strong sleep latency increase, and decreased sleep. If astrocytic GAT regulates GABAergic tone on 1-LNvs, we would predict that a null wide-awake mutation would suppress $g a t^{33-1}$ sleep phenotypes. We therefore generated wake ${ }^{d 2}$; gat $^{33-1}$ double-mutants and measured their sleep (Figure 5). wake $^{d 2}$; $g a t^{33-1}$ flies exhibited a dramatic reduction in sleep amounts compared to $g a t^{33-1}$ flies. These double mutants actually slept as little as single wake ${ }^{d 2}$ mutants (Figures 5A and 5B). Similarly, wake ${ }^{d 2}$ was epistatic to gat $^{33-1}$ for the other sleep phenotypes we measured, such as abnormal sleep latency (even at night), sleep frequency and sleep episode length (Figures 5C, 5D and 5E). We did not find any difference in the mean activity per minute while moving between double mutants and controls (Figure 5F). Similar results were obtained with female wake ${ }^{d 2}$; $g a t^{33-1}$ flies (Figures S4A-S4F). We therefore conclude that wake functions downstream of gat to regulate sleep,

\section{Reducing RDL signaling in l-LNvs suppresses gat $^{33-1}$ sleep phenotypes}

The wake-gat epistasis experiments and the physical proximity of GAT positive astrocytes near RDL-positive 1-LNvs suggest that GAT regulates GABAergic signaling in 1-LNvs. However, wake has recently been shown to function not just in 1-LNvs but also in circadian Dorsal Neurons 1 (DN1s) ${ }^{46}$. To definitely map GAT function to GABAergic 1LNv synapses, we first downregulated RDL only in PDF-positive LNv neurons of gat $^{33-1}$ flies, using $p d f$-GAL4, UAS-dicer ${ }^{47}$. The total amount of sleep was reduced in these double-mutant flies compared to $g a t^{33-1}$ and resembled the amount of sleep observed when downregulating RDL in a wild-type background (Figures 6A and 6B). Similar results were observed in female flies (Figures S5A-S5B). Other gat $^{33-1}$ sleep parameters were also corrected (Figures 6C-6F; Figures S5C-S5F), indicating that $r d l$ in PDFpositive circadian neurons is epistatic to gat. In our hands however, $r d l$ knockdown did not show a strong effect on sleep in a wild-type background, contrary to previous reports 
${ }^{28,30}$. We presume that this is because of a weaker $r d l$ knockdown. Thus, to further support the idea that GAT regulates GABA signaling in 1-LNvs, we downregulated the RDL modulator Neuroligin-4 (DNLG4) in PDF-positive neurons of gat $^{33-1}$ mutant flies. DNlg4 is a synaptic adhesion molecule and promotes sleep by facilitating RDL membrane clustering in I-LNvs, thereby promoting GABA signaling ${ }^{32}$. Again, we observed that $d n l g 4$ downregulation in PDF neurons suppressed the gat $^{33-1}$ sleep phenotypes in males and females (Figures 7A-7G; Figure S6A-S6G). GAT thus functions upstream of DNlg4 in PDF-positive circadian neurons, and the GAT sleep phenotype is largely, if not entirely, caused by dysregulation of GABA signaling onto PDF neurons. Interestingly, dnlg4 downregulation only significantly reduces sleep during the night ${ }^{32}$ but suppress the GAT sleep phenotypes during the day as well.

Combined with prior studies ${ }^{24,27,28,30,31,32}$, all our imaging and genetic epistasis experiments point to astrocytic GAT regulating sleep through reuptake of GABA near PDF-positive 1-LNvs.

\section{Discussion:}

The transition between sleep and wakefulness and thereafter maintenance of sleep and wake stage requires a fine synchronization of various distinct neuronal signaling controlled by circadian and homeostatic processes ${ }^{13,23,48}$. While the circadian clock regulates the timing of sleep onset and arousal, homeostatic processes respond to sleep pressure buildup by increasing sleep. Homeostasis can override circadian control when sleep pressure is high ${ }^{12,49}$. Several neurotransmitters orchestrate signaling between sleep, arousal and circadian neurons to achieve this plasticity ${ }^{6,50}$. GABA has been typically considered as a sleep-promoting neurotransmitter ${ }^{51,52}$. Genetic and pharmacological manipulations of brain GABAergic neurons have similar impact on sleep in Drosophila and mammals, indicating a conserved mechanism of action. Several drugs target GABA receptors, thus boosting GABA signaling and enhancing sleep ${ }^{53,54,55}$. In addition to neurons, astrocytes are actively engaged in controlling sleep and clearing out metabolic waste products ${ }^{56}$. A recent study reports that calcium level in astrocytes changes significantly during the sleep-wake transition ${ }^{57}$, highlighting its indispensable role in sleep control.

In our study, we observed that modulation of GABA signaling by the astrocytic GABA transporter GAT is crucial to the proper regulation of Drosophila sleep. We relied on a novel and viable hypomorphic gat mutant that revealed this gene's critical sleep function. gat $^{33-1}$ mutant showed increased sleep amount and consolidation. This is consistent with findings in the mammalian system where GAT-1 deletion caused sleep disturbances and altered sleep architecture in mice ${ }^{58,59}$. Moreover, neuropathic pain activates astrocytes and increases astrocytic GAT-3 expression in the anterior cingulate cortex, thus 
decreasing sleep ${ }^{60}$. We found that in Drosophila GAT regulates sleep mostly, if not exclusively, by controlling GABAergic tone on the wake-promoting I-LNvs. This is mediated through the $\mathrm{GABA}_{\mathrm{A}}$ receptor RDL present on I-LNvs. Indeed, our epistasis experiments show that GAT functions upstream of RDL to regulate sleep. The transaminase GABAT probably helps reducing GABA tone downstream of GAT ${ }^{61}$.

Upon binding to RDL, GABA promotes sleep by reducing the electrical activity of the ILNvs. Importantly, RDL abundance at the plasma membrane is rhythmically regulated by the circadian clock. This is achieved through circadian regulation of WAKE, which promotes trafficking of RDL to the plasma membrane ${ }^{31}$, and circadian regulation of RDL degradation by FBXL4 ${ }^{33}$. Reduction in GAT activity slows down GABA removal, resulting in more sustained RDL activation. This results in increased sleep even when membrane RDL expression is decreased. Our findings thus show the key contribution of the astrocytes in shaping the circadian regulation of sleep by limiting GABA tone, and thus presumably amplifying the importance of RDL rhythms.

Overall, our observations are remarkably consistent in supporting astrocytic GAT's impact on both daytime and night-time sleep. However, we noted that astrocyte-specific GAT expression did not completely restore sleep amount at night. This might be because we did not accurately restored GAT activity levels with alrm-GAL4. More surprising perhaps was that we did not restore sleep latency at night, not even with a genomic construct that tended to overcompensate gat $^{33-1}$ phenotypes. However, the latency phenotype was suppressed by all our genetic manipulation affecting GABA signaling, which would actually support a role for GAT in sleep-night latency. Night-time sleep latency might thus be particularly sensitive to accurate GAT activity level, and neither rescue approaches provided it.

While our results strongly support a key role for GAT in regulating the circadian 1-LNvs and thus the timing and amount of sleep, GAT might also play a role in sleep homeostasis Indeed, we observed GAT-positive astrocytes throughout the adult brain (video-S5). A previous study found that the fan-shaped body (dFB) of the central complex is part of the Drosophila sleep homeostat. dFB releases GABA to inhibit octopaminergic arousal neurons and promotes sleep ${ }^{23}$. Abundant protein expression of RDL and GABergic input is also present on R-neurons of the ellipsoid body $(\mathrm{EB})$ in the central complex ${ }^{62}$.

Moreover, GABAergic control of wake-promoting dopaminergic signaling in the central brain was detected ${ }^{63}$. Future studies will be needed to determine whether GAT plays a role in sleep homeostasis or influence sleep in other manners than through the 1-LNvs.

In conclusion, our work provides direct support for astrocyte-dependent sleep regulation through GAT, whereby GAT regulates GABA tone on circadian I-LNvs. These insights 
could prove important to understand and improve treatment of sleep deficits, since GAT is a well-defined pharmacological target.

\section{Acknowledgement:}

We are grateful to the Vienna Drosophila RNAi Center and the Bloomington Stock Center for providing fly stocks. We are thankful to Prof. R. L. Davis, Scripps Research Florida for providing anti-RDL antibody, and Prof. R. Stanewsky, Münster University, Germany, for anti-PER antibody. Anti-PDF, anti-GFP, anti-Pros and anti-Spectrin were obtained from the Developmental Studies Hybridoma Bank, USA. We are grateful to Prof. Mark Wu, Johns Hopkins University, USA, for wake mutant flies and Prof. Junhai Han, Southeast University Nanjing, China for the UAS-dnlg4-RNAi fly line. We also thank Prof. Leslie Griffith and Patricia Goodwin at Brandeis University, USA, for helping us setting up sleep assays in our ab. We thank all members of the Emery, Weaver and Anaclet lab for insightful discussions on the work and manuscript. This work was supported by a MIRA award from the National Institute of General Medicine Sciences (1R35GM118087) to P.E. and NIH RO1 NS053538 to M.F.

\section{Author Contributions:}

Conceptualization, R.C. and P.E.,

Methodology, R.C., T.S., and C.Y.

Investigation, R.C., C.Y. and T.S.

Writing -Original Draft, R.C. and P.E.

Writing- Review \& Editing, T.S. and M.F.

Funding Acquisition, M.F. and P.E.

Supervision, P.E. and M.F.

\section{Declaration of Interests:}

The authors declare no competing interests.

\section{Figure titles and legends:}

Figure 1. gat $^{33-1}$ is a hypomorphic mutant of GABA transporter (GAT):

(A) Schematic of the gat gene locus. TALEN targeting site in the first exon is indicated.

(B) Nucleotide sequence of the targeted region of gat. The grey boxes indicate the target sites for TALEN-mediated mutagenesis in the $1^{\text {st }}$ exon, the start codon is indicated in green. gat $^{33-1}$ mutants harbor a 7 nucleotides deletion that removes a Bcc1 endonuclease restriction site and leads to a frameshift and early termination of the GAT protein. (C) Protein sequence of the GAT N-terminus. Amino acids encoded by exon 1 and 2 are indicated in orange and blue respectively. The beginning of the first transmembrane domain (TM) is indicated by a light blue box. The frameshift in gat $^{33-1}$ should lead to an 
early termination after 20 amino acids (red), however downstream methionines (Green boxes) could act as alternative start sites. (D) Schematic overview of the putative GAT membrane topology. The sites of the two putative alternative start sites in gat $^{33-1}$ mutants are indicated (Blue arrows) and they would lead to 22 aa or 64aa truncations of the cytoplasmic N-terminus. (E) Western blot analysis of GAT protein of wild type and gat $^{33-}$ ${ }^{l}$ mutant fly heads using $\mathrm{C}$ - and N-terminal anti-GAT antibodies. The C-terminal antiGAT antibody recognizes a strongly reduced and truncated GAT specific band (white arrow) in $g a t^{33-1}$ mutant extracts while the $\mathrm{N}$-terminal anti-GAT antibody only recognized a GAT specific band in wild type control flies (black arrow). (F) Quantification of GAT and PER levels at different timepoints during the day and night. Zeitgeber Time (ZT) 0 correspond to lights-on and ZT12 to lights-off transitions, $(n=3)$. The quantified protein level was normalized to Spectrin levels. (G) Circadian period lengths (two tailed t-test) and $(\mathbf{H})$ circadian rhythmicity (Fisher's extract test) of $g a t^{33-1}(\mathrm{n}=60)$ and control $w^{1118}$ $(n=63)$ male flies (error bars represent SEM).

\section{Figure 2. GABA transporter (GAT) is required for normal sleep-wake behavior}

(A) Sleep profiles of gat $^{33-1}$ (green, $\mathrm{n}=110$ ) and control $w^{1118}$ (black, $\mathrm{n}=93$ ) male flies in $12 / 12 \mathrm{~h}$ LD cycle showing enhanced amount of sleep in the gat hypomorphic mutant during both day and night. (B-E) Total sleep amounts (min) (B), number of sleep episodes (C), mean sleep episode's durations ( $\mathrm{min}$ ) (D), and mean wake episode's durations ( $\mathrm{min}$ ) (E) in 24-hours (h) period, during daytime, and during night showing increase in sleep consolidation in $g t^{33-1}$. (F) Latency to sleep (min) during the day and nighttime indicates accelerated sleep initiation in gat $^{33-1}$. (G) Sleep profile of gat $^{33-1}$ (green, $\mathrm{n}=32$ ) and control $w^{1118}$ (black, $\mathrm{n}=32$ ) male flies in constant darkness (DD). Results show that enhancement of gat $^{33-1}$ sleep is not light dependent. (H) Total amount of sleep during DD. (I) Mean activity per min while moving during DD. (J) Mean activity per min while moving during LD cycle. Error bar represents SEM. For normally distributed data, a two-tailed Student's t-test was used. In case of non-gaussian distribution, a Mann-Whitney U-test was performed, and statistical significance is shown as $* * * \mathrm{P}<0.001 ; * * \mathrm{P}<0.01$; and 'ns' as not significant.

\section{Figure 3. gat $^{33-1}$ sleep defects are rescued by expressing gat in astrocytes}

(A) Sleep profile of male flies with genotype gat $^{33-1}$ (green, $\mathrm{n}=90$ ), control $w^{1118}$ (black,

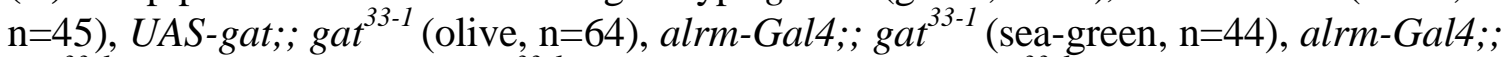
gat $^{33-1} /$ UAS-genomic-gat; ; gat $^{33-1}$ (red, $\mathrm{n}=96$ ), and 1b12;; gat $^{33-1}$ (crimson, $\mathrm{n}=37$ ) in 12/12 $\mathrm{h}$ LD cycle. The results show that GAT functions in astrocytes. (B-D) Total sleep amounts (min) (B), mean sleep episode's durations (min) (C), and number of sleep episodes (beam breaks) (D) during 24-hours (h) period, day, and night. (E) Latency to sleep (min) during day, and nighttime. (F) Mean activity per min while moving per 24hour (h), day, and night. Error bar represents SEM. For normally distributed data, oneway ANOVA followed by a Tukey post-hoc test was performed. For non-gaussian distribution, a Kruskal-Wallis test with Dunn's post-hoc test was performed. Different alphabetical letters (a, b, c, and d) illustrate statistically significant differences between 
groups. The same statistical tests were used in Figures 5-7, as well as Supplemental Figures S3, S4 and S5.

\section{Figure 4. RDL and PDF expressing wake promoting clock neurons (I-LNvs) are in} close proximity with GAT expressing astrocytes.

(A) Confocal analysis of $w^{1118}$ wild type fly brains for localization of RDL (blue) in PDF (red) positive 1-LNvs. Astrocytes are expressing membrane bound GFP (green) (alrmGal4>UAS-CD8-GFP). (a-a3) Labeling in large ventral lateral neurons (I-LNvs). (b-b3) Labeling in medulla region of optic lobe. 1-LNvs neurons also extend its projections in the medulla. (c) 3D view of image-stacks demonstrate projections of astrocytes (green) are extending towards /close to the cell body of l-LNvs and co-localized with RDL, and PDF. (B) Magnified view of 1-LNvs labeled with RDL (blue) (a), astrocytes (green) (b), PDF (red) in l-LNvs (c). Merged image (d) was used for orthogonal projection (d' and d") where yellow arrows point to astrocytes situated very closely to RDL, and PDF expressing in 1-LNvs. (C) Magnified view of themedulla region in the optic lobe labeled for RDL (blue) (a), astrocytes (green) (b), and PDF (red) (c). Merged image (d) was used for orthogonal projections (d' and d") where yellow arrows point to astrocytes processes approaching RDL and PDF positive l-LNvs projections innervating into medulla. (D) Confocal analysis of $w^{1118}$ brains for localization of GAT (blue), PDF positive circadian neurons (red), and astrocytes expressing membrane bound GFP (green) (alrmGal4>UAS-CD8-GFP). (a-a3) Labeling in large ventral lateral neurons (I-LNvs). (b-b3) Labeling in the medulla region of the optic lobe. (E) Magnified view of 1-LNvs region labeled with GAT (blue) (a), astrocytes (green) (b), PDF (red) in 1-LNvs (c). Merged image (d) was used for orthogonal projection (d' and d") where yellow arrows point to GAT present in astrocytes and closely placed to PDF expressing in 1-LNvs. (F) Magnified view of the medulla region of the optic lobe labeled for GAT (blue) (a), astrocytes (green) (b), and PDF (red) (c). Merged image (d) was used for orthogonal projections (d' and d") where yellow arrows point to GAT in astrocytes processes approaching to PDF positive 1-LNvs. Scale bars are represented in respective image panels.

\section{Figure 5. A wide wake (wake) mutation suppresses gat $^{33-1}$ sleep phenotypes}

(A) Sleep profile of male flies with genotype gat $^{33-1}$ (green, $\mathrm{n}=18$ ), control $w^{1118}$ (black, $\mathrm{n}=24$ ), wide-awake mutant wake $^{d 2}$ (orange, $\mathrm{n}=22$ ), and double-mutant with wake $^{d 2} ;$ gat $^{33-}$ ${ }^{1}$ (pink, $\mathrm{n}=19$ ) in 12/12 h LD cycle. The results show that excessive sleeping in gat $^{33-1}$ mutants is suppressed in the double-mutant flies (B) Quantification of total sleep amounts (min) over 24 hours (h), during day, and during night. (C) Quantification of mean sleepepisode's durations lengths (min). (D) Number of sleep episodes indicates fragmented sleep in wake $e^{d 2}$ and double-mutant flies. (E) Higher latency to sleep (min) shows difficulty in initiation of sleep in wake $e^{d 2}$ and double-mutant flies compared to gat $^{33-1}$ and $w^{1118}$ controls. (F) Mean activity per min while moving during 24-hour (h) period, day, and night did not show any significant difference between wake $e^{d 2}$ flies, double-mutants and controls. Error bar represents SEM. Statistics as in figure 3 
Figure 6. GABA $\mathrm{A}_{\mathrm{A}}$ receptor RDL depletion in PDF neurons suppresses the gat $^{33-1}$ sleep phenotypes.

(A) Sleep profile of male flies with genotype PD2-Gal4;; gat $^{33-1}$ (brown, $\mathrm{n}=70$ ), UAS$r d l^{R N A i} ; ; g a t^{33-1}$ (navy blue, $\mathrm{n}=42$ ), PD2-Gal4;;gat ${ }^{33-1}>U A S-r d l^{R N A i} ; ; g a t^{33-1}$ (red, $\mathrm{n}=53$ ), and PD2-Gal4>UAS-rdl ${ }^{R N A i}$ (lavender, $\mathrm{n}=33$ ) in 12/12 h LD cycle. The results show that excessive sleeping in $g a t^{33-1}$ mutants is suppressed by $r d l$ RNAi knockdown in PDF neurons (B) Quantification of total sleep amount (min) over 24 hours (h), during day, and during night. (C) Quantification of mean sleep episode's durations (min). (D) Number of sleep episodes indicate fragmented sleep in RDL knockdown flies with or without gat $^{33-1}$. (E) Latency to sleep (min) in RDL knockdown flies with and without gat $^{33-1}$ (F) Mean activity per min while moving during 24-hour (h) period, day, and night. Error bar represents SEM. Statistics as in Figure 3.

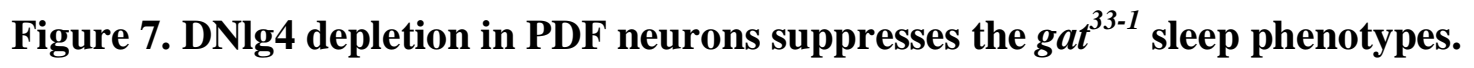

(A) Sleep profile of male flies with genotype gat $^{33-1}$ (green, n=15), PD2-Gal4;; gat $^{33-1}$

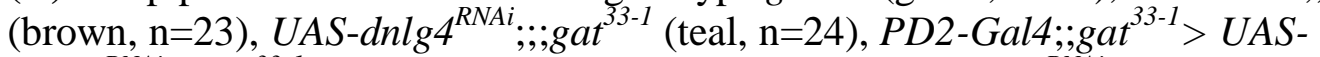

$\operatorname{dnlg} 4^{R N A i} ; ; ; g^{2 a t}{ }^{33-1}$ (purple, $\mathrm{n}=23$ ), and PD2-Gal4>UAS-dnlg $4^{R N A i}$ (blue, $\mathrm{n}=24$ ) in 12/12 h LD cycle. The results show that excessive sleeping in gat $^{33-1}$ mutants is suppressed during nighttime by dnlg4 RNAi knockdown in PDF neurons (B) Quantification of total sleep amounts ( $\mathrm{min}$ ) over 24 hours (h), during day, and during night. Results show reduction in sleep during night in $d n l g 4$ knockdown flies with and without $g a t^{33-1}$. (C) Quantification of mean sleep-episode's durations (min). (D) Number of sleep episodes. (E) Maximum sleep episode's duration ( $\mathrm{min}$ ). (F) Higher latency to sleep ( $\mathrm{min}$ ) shows difficulty in initiation of sleep in $d n l g 4$ knockdown flies with and without $g a t^{33-1}$. (G) Mean activity per min while moving during 24-hour (h) period, day, and night. Error bar represents SEM. Statistics as in Figure 3.

\section{Materials and Methods:}

\section{Fly Strains and Rearing Conditions:}

All flies were reared on standard cornmeal/agar medium supplemented with yeast. The following Drosophila strains were used: $p d f$-GALA, UAS-dicer $2 / C y O(P D 2)^{47} ; p d f$ GAL4 (PG4) ${ }^{45}$. alrm-GAL4 ${ }^{35} ;$ Df(4)gat ${ }^{464 k b}, U A S$ - gat (contains introns), and $1 b 12^{37}$. $r d l$ RNAi line (GD, stock no. v41103) and $w^{1118}$ flies were obtained from the Vienna Drosophila Stock Center. wake $e^{d 2}$ flies were a generous gift from Mark Wu at Johns Hopkins University, USA ${ }^{31}$. UAS- $\ln \lg 4^{R N A i}$ was a very kind gift from Junhai Han, Southeast University Nanjing, China ${ }^{32}$. UAS-mCD8::GFP was obtained from Bloomington Stock Center, USA. The following flies were generated for this study: GAT (CG1732) mutant $\left(\mathrm{gat}^{33-1}\right), 1 \mathrm{b12} ; \mathrm{gat}^{33-1}$, wake ${ }^{d 2} ; \mathrm{gat}^{33-1}, \mathrm{UAS}-\mathrm{dnlg} 4^{R N A i}$;; $\mathrm{gat}^{33-1}$, PD2;; gat $^{33-1}, U A S-r d l^{R N A i} ; g a t^{33-1} \cdot$ gat $^{33-1}$, alrm-Gal4, UAS-gat, and wake ${ }^{d 2}$ flies were backcrossed into the $w^{1118}$ genetic background for 6 generations.

\section{Generation of gat $^{33-1}$ hypormorphic mutant:}


gat $^{33-1}$ hypomorphic mutants were generated using a TALEN approach as described in Stork et al.,2014 ${ }^{37}$. In short TALEN encoding mRNAs targeting the coding region of the first exon of gat were injected into early embryos. Subsequently mutants in gat were identified by a combination of restriction digests of an amplicon covering the targeted region as well as complementation analysis using the gat locus deficiency $D f(4) g^{4 t}{ }^{464 k b}$ 37 .

\section{Sleep measurement and analysis:}

Sleep experiments were carried out with both adult male and mated female flies (3-5 days old) separately. Individual flies were introduced into $65 \mathrm{~mm}$ glass tubes containing agar (2\%) and sucrose (5\%). These tubes were loaded into Drosophila Activity Monitors (DAM, Trikinetics system, Waltham, MA), and locomotor activity measured every minute. The experiments were conducted in 136-LL Percival incubators (Percival Scientific, Perry IA) under a light-dark cycle (LD; $12: 12 \mathrm{~h}$ ) or constant darkness at $25^{\circ} \mathrm{C}$ or $29^{\circ} \mathrm{C}$. Flies were exposed for 6 days to a standard light/dark cycle (LD, $12 \mathrm{~h} / 12 \mathrm{~h}$ ). Sleep was analyzed between days 4- 6 and averaged. For some experiments, flies were then exposed to constant darkness as well. Using DAMsystem3 and DAMfilescan111 software (TriKineticsDAMSystem), data files (30 min and $1 \mathrm{~min}$ ) were generated for Sleep and Circadian Analysis MATLAB Program (SCAMP) (MathWorks, Natick, MA). (http://www.bio.brandeis.edu/tracker/SleepAnalysis/SleepAnalysisInstructions.pdf) ${ }^{64}$. A minimum 5 min period of inactivity (defined as no beam breaks in DAM) was considered as sleep. Sleep profile (sleep time/30 min), sleep frequency (no of sleep episodes per 24 $\mathrm{h}$, day and night), mean sleep episode duration (bout length), sleep latency [time taken to fall asleep after the light goes off (night) or turns on (day)], maximum sleep episode duration, maximum wake episode duration, mean wake episode duration, and mean activity per min while awake were calculated from SCAMP.

\section{Circadian Behavior:}

Male flies were entrained for 5 days to an $\mathrm{LD}$ cycle $(12: 12 \mathrm{~h})$ at $25^{\circ} \mathrm{C}$, followed by 6 days of constant darkness. Locomotor activity was measured as described in the sleep experiment section with the DAM system. Data analysis was performed with the FAASX software (https://neuropsi.cnrs.fr/en/cnn-home/francois-rouyer/faas-software/, M. Boudinot and F. Rouyer, Centre National de la Recherche Scientifique, Gif-sur-Yvette Cedex, France). Three independent experiments were averaged for period length and rhythmicity. Actogram and Education graphs were also generated with FAAS-X.

\section{Protein Extraction, Western blot, and Quantification:}

20 fly heads were collected for each sample, homogenized and lysed in RIPA buffer containing protease inhibitors cocktail (PMSF, leupeptin, pepstatin A, aprotinin). The samples were shaken at $4^{\circ} \mathrm{C}$ for $30 \mathrm{~min}$ and then spun at $13000 \mathrm{~g}$ for $10 \mathrm{~min}$ to remove 
insoluble material. Without boiling, samples were mixed with SDS-PAGE sample buffer (100mM Tris, pH 6.8, 4.4\% SDS, glycerol, 100mM DTT, and 0.04\% bromophenol-blue) and resolved by SDS-PAGE. Western Blots were stained with rabbit anti-GAT Nterminus, rabbit anti-GAT C-terminus (both 1:10000) ${ }^{37}$, mouse anti-PER (1:10000) generous gift from Dr. R. Stanewky), and mouse anti-Spectrin (1:5000) from Developmental Studies Hybridoma Bank (DSHB, 3A9). Secondary antibodies conjugated with HRP from Jackson Immuno Research were used at 1:10,000 dilutions. For quantification, western blot bands were analyzed using Image $\mathbf{J}$ (http://rsb.info.nih.gov/ij). GAT and PER protein levels were normalized with Spectrin levels. For the truncated GAT protein in gat $^{33-1}$ mutant, near infrared western blot detection method was used (LI-COR Biosciences). After transfer membranes were blocked in intercept buffer (Li-COR, cat \# P/N 927-60001) for 1 hour at room temperature with gentle shaking. Blots were incubated with rabbit anti-GAT N-terminus or rabbit anti-GAT C-terminus (both, 1:5000) diluted in intercept buffer containing $0.2 \%$ Tween 20 for overnight at $4{ }^{\circ} \mathrm{C}$. The bands were detected with secondary antibody, IRDye 800CW (LI-COR, cat \# P/N 925-32211) (1:10,000) using LI-COR Odyssey Infrared Imaging System. Each experiment was performed thrice and showed reproducible results.

\section{Brain Dissection, and Immunostaining:}

Adult brain: Fly heads were removed from the body and brains were dissected in PTX (0.1\% Tx-100, 1XPBS). Dissected brains were fixed in 4\% paraformaldehyde for 20 min followed by 5 washes with PTX $(0.3 \%$ Tx-100, 1XPBS) and then 3 washes with PBT (0.1\% BSA, $0.3 \%$ Tx-100, 1XPBS) for 5-10 min per wash. The brains were blocked in PBT for $2 \mathrm{~h}$ at room temperature and then incubated with primary antibodies at $4^{\circ} \mathrm{C}$ for 2 nights. After 6 washes for 10 min each with PBT, samples were probed with appropriate secondary antibodies overnight at $4^{\circ} \mathrm{C}$, washed 6 times for 10 min each with PBT, and then mounted in Vectashield anti-fade mounting medium (Vector Laboratories). Primary antibodies used were chicken anti-GFP antibody (ab13970; 1:1,000; Abcam), mouse antiPDF antibody (1:400; Developmental Studies Hybridoma Bank DSHB, C7), rabbit antiRDL (1:800) and rabbit anti-GAT C-terminus (1:5000). Secondary antibodies used were Alexa Fluor 488-conjugated anti-chicken, Alexa Fluor 555-conjugated anti-rabbit, and Alexa Fluor 635-conjugated anti-mouse (Molecular Probes) at 1:500 dilution. Mounted brains were scanned using a Zeiss LSM5 Pascal confocal microscope. The brains were scanned for Z-stack and orthogonal projections were used to visualize protein (GAT, PDF, RDL and GFP in astrocytes) localization.

Larval brains: A protocol similar to adult brain was used, with the following differences. Dissection buffer was PBS (pH 7.2), PTX contained 0.2\% Tx-100. A 1h blocking in 1\% BSA was performed in PTX. Primary antibodies were anti-GAT C-terminus (1:5000 dilution) and anti-PROS (DSHB, MR1A, 1:150 dilution), secondary antibodies were 
donkey antibodies from Jackson ImmunoResearch AlexaFluor-488 conjugated antimouse (715-545-151) and Rhodamine Red-X conjugated anti-rabbit (711-295-152). Brains were mounted in Citiflor CFM-1 Plus (Electron Microscopy Sciences) and analyzed on an Innovative Imaging Innovations (3i) spinning-disc confocal microscope equipped with a Yokogawa CSX-X1 scan head and analyzed using Slidebook software (3i).

\section{Statistical analysis:}

GraphPad Prism 9.0 was used to plot graphs and compare independent groups of data. The graph represents the means of \pm S.E.M. data pooled from at least three independent sets of experiments (technical replicates) and ' $n$ ' represents the biological replicates or total numbers of flies. Male and female sleeps were analyzed separately. All the data were tested for normality using the D'Agostino and Pearson omnibus test. In the case of normally (Gaussian) distributed data, a two-tailed Student's t-test was used for two groups and one-way ANOVA followed by a Tukey post hoc test was performed for multiple groups. For non-Gaussian distribution, a Mann-Whitney U-test was performed for two groups, while for multiple groups, the Kruskal-Wallis test with Dunn's post-hoc test was performed. Statistical significance is demonstrated as $* * * * \mathrm{P}<0.0001$; ***P < $0.001 ; * * \mathrm{P}<0.01 ; * \mathrm{P}<0.05$. For multiple groups, the same alphabetical letters indicate that two groups are not significantly different.

\section{References:}

1. Miyazaki, S., Liu, C.Y., and Hayashi, Y. (2017). Sleep in vertebrate and invertebrate animals, and insights into the function and evolution of sleep. Neuroscience Research 118 , $3-12$.

2. Keene, A.C., and Duboue, E.R. (2018). The origins and evolution of sleep. The Journal of experimental biology 221 (Pt 11), jeb159533.

3. Anafi, R.C., Kayser, M.S., and Raizen, D.M. (2019). Exploring phylogeny to find the function of sleep. Nature Reviews Neuroscience 20 (2), 109-116.

4. Ungurean, G., van der Meij, J., Rattenborg, N.C., and Lesku, J.A. (2020). Evolution and plasticity of sleep. Current Opinion in Physiology 15, 111-19.

5. Sehgal, A., and Mignot, E. (2011). Genetics of sleep and sleep disorders. Cell 146 (2), 194-207.

6. Ly, S., Pack, A.I., and Naidoo, N. (2018). The neurobiological basis of sleep: Insights from Drosophila. Neuroscience and Biobehavioral Reviews 87, 67-86. 
7. Pace-Schott, E.F., and Hobson, J.A. (2002). The neurobiology of sleep: Genetics, cellular physiology and subcortical networks. Nature Reviews Neuroscience 3 (8), 591-605.

8. Artiushin, G., and Sehgal, A. (2020). The Glial Perspective on Sleep and Circadian Rhythms. Annual Review of Neuroscience 43, 119-40.

9. Garofalo, S., Picard, K., Limatola, C., Nadjar, A., Pascual, O., and Tremblay, M.È. (2020). Role of glia in the regulation of sleep in health and disease. Comprehensive Physiology 10 (2), 687-712.

10. Jackson, F.R., You, S., and Crowe, L.B. (2020). Regulation of rhythmic behaviors by astrocytes. Wiley Interdisciplinary Reviews: Developmental Biology 9 (4), e372.

11. Jackson, F.R., Ng, F.S., Sengupta, S., You, S., and Huang, Y. (2015). Glial cell regulation of rhythmic behavior. In Methods in Enzymology 552, 45-73.

12. Borbely, A.A. (1982). A two process model of sleep regulation. Human Neurobiology 1 (3), 195-204.

13. Borbély, A.A., Daan, S., Wirz-Justice, A., and Deboer, T. (2016). The two-process model of sleep regulation: A reappraisal. Journal of Sleep Research 25 (2), 131-43.

14. Scammell, T.E., Arrigoni, E., and Lipton, J.O. (2017). Neural Circuitry of Wakefulness and Sleep. Neuron 93 (4), 747-65.

15. Potdar, S., and Sheeba, V. (2018). Wakefulness is promoted during day time by PDFR signalling to dopaminergic neurons in Drosophila melanogaster. eNeuro 5 (4), ENEURO.0129-18.2018.

16. Roth, F.C., and Draguhn, A. (2012). GABA metabolism and transport: effects on synaptic efficacy. Neural plasticity $2012,805830$.

17. Wong, C.G., Bottiglieri, T., and Snead III, O.C. (2003). GABA, gamma-hydroxybutyric acid, and neurological disease. Annals of Neurology 54 Suppl 6, S3-12.

18. Brickley, S.G. and I.Mody. (2012). Extrasynaptic GABA(A) receptors: Their function in the CNS and implications for disease. Neuron 73(1), 23-34.

19. Jacob, T.C., Moss, S.J., and Jurd, R. (2008). GABA(A) receptor trafficking and its role in the dynamic modulation of neuronal inhibition. Nature Reviews Neuroscience 9 (5), 33143.

20. Michels, G., and Moss, S.J. (2007). GABAA receptors: Properties and trafficking. Critical Reviews in Biochemistry and Molecular Biology 42 (1), 3-14.

21. Franco, D.L., Frenkel, L., and Ceriani, M.F. (2018). The underlying genetics of Drosophila circadian behaviors. Physiology 33 (1), 50-62.

22. Liu, C., and Reppert, S.M. (2000). GABA synchronizes clock cells within the suprachiasmatic circadian clock. Neuron 25 (1), 123-8. 
23. Ni, J.D., Gurav, A.S., Liu, W., Ogunmowo, T.H., Hackbart, H., Elsheikh, A., Verdegaal, A.A., and Montell, C. (2019). Differential regulation of the drosophila sleep homeostat by circadian and arousal inputs. eLife 8, e40487.

24. Parisky, K.M., Agosto, J., Pulver, S.R., Shang, Y., Kuklin, E., Hodge, J.J.L., Kang, K., Liu, X., Garrity, P.A., Rosbash, M., et al. (2008). PDF Cells Are a GABA-Responsive Wake-Promoting Component of the Drosophila Sleep Circuit. Neuron 60 (4), 672-82.

25. Ono, D., Honma, K. ichi, Yanagawa, Y., Yamanaka, A., and Honma, S. (2018). Role of GABA in the regulation of the central circadian clock of the suprachiasmatic nucleus. Journal of Physiological Sciences 68 (4), 333-43.

26. Sheeba, V., Fogle, K.J., Kaneko, M., Rashid, S., Chou, Y.T., Sharma, V.K., and Holmes, T.C. (2008). Large Ventral Lateral Neurons Modulate Arousal and Sleep in Drosophila. Current Biology 18 (20), 1537-45.

27. Shang, Y., Griffith, L.C., and Rosbash, M. (2008). Light-arousal and circadian photoreception circuits intersect at the large PDF cells of the Drosophila brain.

Proceedings of the National Academy of Sciences of the United States of America 105 (50), 19587-94.

28. Chung, B.Y., Kilman, V.L., Keath, J.R., Pitman, J.L., and Allada, R. (2009). The GABAA Receptor RDL Acts in Peptidergic PDF Neurons to Promote Sleep in Drosophila. Current Biology 19 (5), 386-90.

29. Gmeiner, F., Kolodziejczyk, A., Yoshii, T., Rieger, D., Nässel, D.R., and Helfrich-Förster, C. (2013). GABA(B) receptors play an essential role in maintaining sleep during the second half of the night in Drosophila melanogaster. Journal of Experimental Biology 216 (Pt 20), 3837-43.

30. Agosto, J., Choi, J.C., Parisky, K.M., Stilwell, G., Rosbash, M., and Griffith, L.C. (2008). Modulation of GABAA receptor desensitization uncouples sleep onset and maintenance in Drosophila. Nature Neuroscience 11 (3), 354-9.

31. Liu, S., Lamaze, A., Liu, Q., Tabuchi, M., Yang, Y., Fowler, M., Bharadwaj, R., Zhang, J., Bedont, J., Blackshaw, S., et al. (2014). WIDE AWAKE mediates the circadian timing of sleep onset. Neuron 82 (1), 151-66.

32. Li, Y., Zhou, Z., Zhang, X., Tong, H., Li, P., Zhang, Z.C., Jia, Z., Xie, W., and Han, J. (2013). Drosophila neuroligin 4 regulates sleep through modulating GABA transmission. Journal of Neuroscience 33 (39), 15545-54.

33. Li, Q., Li, Y., Wang, X., Qi, J., Jin, X., Tong, H., Zhou, Z., Zhang, Z.C., and Han, J. (2017). Fbxl4 Serves as a Clock Output Molecule that Regulates Sleep through Promotion

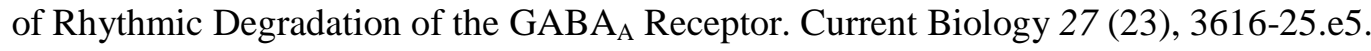

34. Frank, M.G. (2019). The Role of Glia in Sleep Regulation and Function. In Handbook of Behavioral Neuroscience 253, 83-96.

35. Freeman, M.R., and Doherty, J. (2006). Glial cell biology in Drosophila and vertebrates. Trends in neurosciences 29, 82-90. 
36. Muthukumar, A.K., Stork, T., and Freeman, M.R. (2014). Activity-dependent regulation of astrocyte GAT levels during synaptogenesis. Nature Neuroscience 17 (10), 1340-50.

37. Stork, T., Sheehan, A., Tasdemir-Yilmaz, O.E., and Freeman, M.R. (2014). Neuron-Glia interactions through the heartless fgf receptor signaling pathway mediate morphogenesis of drosophila astrocytes. Neuron 83 (2), 388-403.

38. Ransom, C.B., and Richerson, G.B. (2009). Antiepileptic Drugs: GABA Transporters as Targets of Antiepileptic Drugs. In Encyclopedia of Basic Epilepsy Research, 66-74.

39. van Golde, E.G.A., Gutter, T., and de Weerd, A.W. (2011). Sleep disturbances in people with epilepsy; prevalence, impact and treatment. Sleep Medicine Reviews 15 (6), 357-68.

40. Minecan, D., Natarajan, A., Marzec, M., and Malow, B. (2002). Relationship of epileptic seizures to sleep stage and sleep depth. Sleep 25 (8), 899-904.

41. Reddy, D.S., Chuang, S.H., Hunn, D., Crepeau, A.Z., and Magant, R. (2018).

Neuroendocrine aspects of improving sleep in epilepsy. Epilepsy Research 147, 32-41.

42. Elwyn Isaac, R., Li, C., Leedale, A.E., and Shirras, A.D. (2010). Drosophila male sex peptide inhibits siesta sleep and promotes locomotor activity in the post-mated female. Proceedings of the Royal Society B: Biological Sciences 277 (1678), 65-70.

43. Andretic, R., and Shaw, P.J. (2005). Essentials of sleep recordings in Drosophila: Moving beyond sleep time. Methods in Enzymology 393, 759-72.

44. Ho, K.S., and Sehgal, A. (2005). Drosophila melanogaster: An insect model for fundamental studies of sleep. Methods in Enzymology 393, 772-93.

45. Renn, S.C.P., Park, J.H., Rosbash, M., Hall, J.C., and Taghert, P.H. (1999). A pdf neuropeptide gene mutation and ablation of PDF neurons each cause severe abnormalities of behavioral circadian rhythms in Drosophila. Cell 99 (7), 791-802.

46. Tabuchi, M., Monaco, J.D., Duan, G., Bell, B., Liu, S., Liu, Q., Zhang, K., and Wu, M.N. (2018). Clock-Generated Temporal Codes Determine Synaptic Plasticity to Control Sleep. Cell 175 (5), 1213-27.e18.

47. Dubruille, R.L., Murad, A., Rosbash, M., and Emery, P. (2009). A constant light-genetic screen identifies KISMET as a regulator of circadian photoresponses. PLoS Genetics 5 (12), e1000787.

48. Pimentel, D., Donlea, J.M., Talbot, C.B., Song, S.M., Thurston, A.J.F., and Miesenböck, G. (2016). Operation of a homeostatic sleep switch. Nature 536 (7616), 333-7.

49. Donlea, J.M., Pimentel, D., Talbot, C.B., Kempf, A., Omoto, J.J., Hartenstein, V., and Miesenböck, G. (2018). Recurrent Circuitry for Balancing Sleep Need and Sleep. Neuron 97 (2), 378-89.e4.

50. Moszczynski, A., and Murray, B.J. (2012). Neurobiological Aspects of Sleep Physiology. Neurologic Clinics 30 (4), 963-85. 
51. Gottesmann, C. (2002). GABA mechanisms and sleep. Neuroscience 111 (2), 231-9.

52. Anaclet, C., Ferrari, L., Arrigoni, E., Bass, C.E., Saper, C.B., Lu, J., and Fuller, P.M. (2014). The GABAergic parafacial zone is a medullary slow wave sleep-promoting center. Nature Neuroscience 17 (9), 1217-24.

53. Dauvilliers, Y. (2020). Future drugs for sleep and wake disorders. Bulletin de l'Academie Nationale de Medecine 204 (9), 1047-52.

54. Wisden, W., Yu, X., and Franks, N.P. (2019). GABA Receptors and the Pharmacology of Sleep. In Handbook of Experimental Pharmacology 253, 279-304.

55. Sałat, K., and Kulig, K. (2011). GABA transporters as targets for new drugs. Future Medicinal Chemistry 3 (2), 211-22.

56. Haydon, P.G. (2017). Astrocytes and the modulation of sleep. Current Opinion in Neurobiology 44, 28-33.

57. Blum, I.D., Keleş, M.F., Baz, E.S., Han, E., Park, K., Luu, S., Issa, H., Brown, M., Ho, M.C.W., Tabuchi, M., et al. (2021). Astroglial Calcium Signaling Encodes Sleep Need in Drosophila. Current Biology 31 (1), 150-162.e7.

58. Xu, X.H., Qiu, M.H., Dong, H., Qu, W.M., Urade, Y., and Huang, Z.L. (2014). GABA transporter-1 inhibitor NO-711 alters the EEG power spectra and enhances non-rapid eye movement sleep during the active phase in mice. European Neuropsychopharmacology 24 (4), 585-94.

59. Xu, X.H., Qu, W.M., Bian, M.J., Huang, F., Fei, J., Urade, Y., and Huang, Z.L. (2013). Essential Roles of GABA Transporter-1 in Controlling Rapid Eye Movement Sleep and in Increased Slow Wave Activity after Sleep Deprivation. PLoS ONE 8 (10), e75823.

60. Yamashita, A., Hamada, A., Suhara, Y., Kawabe, R., Yanase, M., Kuzumaki, N., Narita, M., Matsui, R., Okano, H., and Narita, M. (2014). Astrocytic activation in the anterior cingulate cortex is critical for sleep disorder under neuropathic pain. Synapse 68 (6):23547.

61. Chen, W.F., Maguire, S., Sowcik, M., Luo, W., Koh, K., and Sehgal, A. (2015). A neuron-glia interaction involving GABA transaminase contributes to sleep loss in sleepless mutants. Molecular Psychiatry 20 (2), 240-51.

62. Xie, X., Tabuchi, M., Brown, M.P., Mitchell, S.P., Wu, M.N., and Kolodkin, A.L. (2017). The laminar organization of the drosophila ellipsoid body is semaphorindependent and prevents the formation of ectopic synaptic connections. eLife 6, e25328.

63. Kim, M., Jang, D., Yoo, E., Oh, Y., Sonn, J.Y., Lee, J., Ki, Y., Son, H.J., Hwang, O., Lee, C., et al. (2017). Rogdi Defines GABAergic Control of a Wake-promoting Dopaminergic Pathway to Sustain Sleep in Drosophila. Scientific Reports 7 (1), 11368. 
64. Donelson, N., Kim, E.Z., Slawson, J.B., Vecsey, C.G., Huber, R., and Griffith, L.C. (2012). High-resolution positional tracking for long-term analysis of Drosophila sleep and locomotion using the "tracker" program. PloS one 7 (5), e37250. 
Figure 1.

A

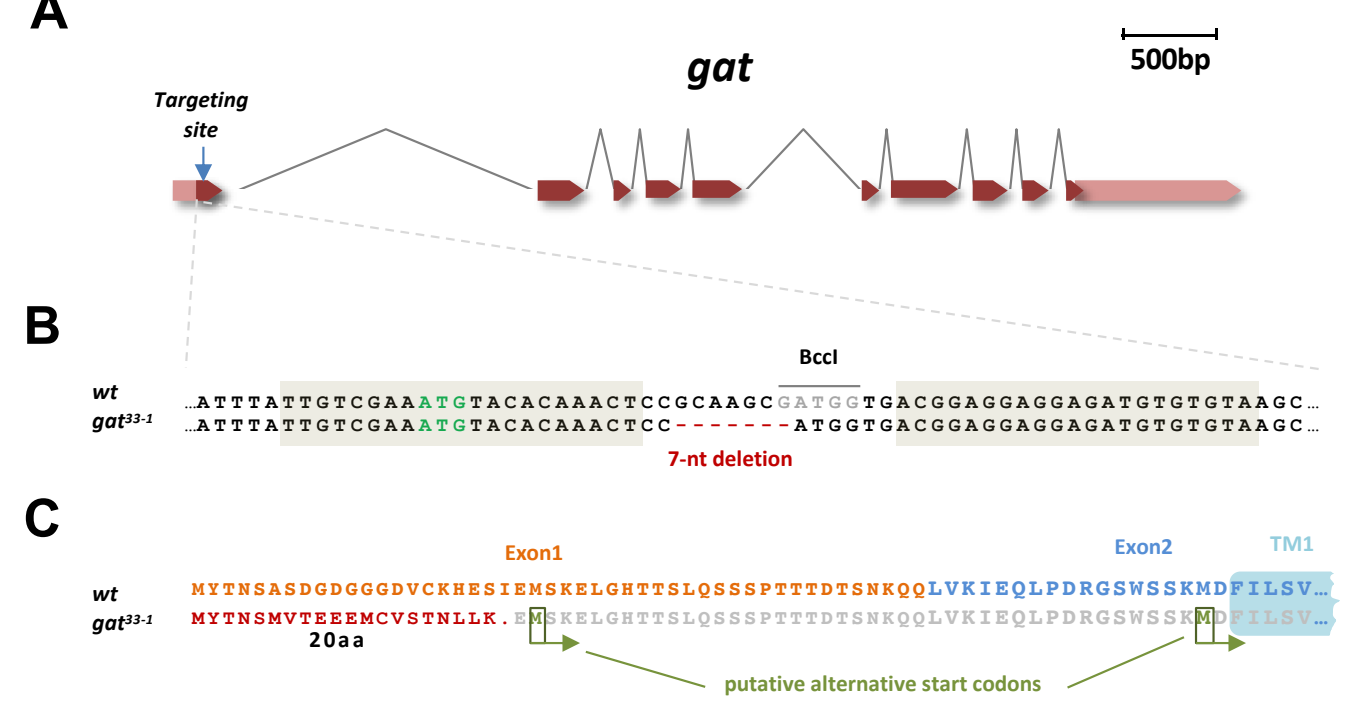

B

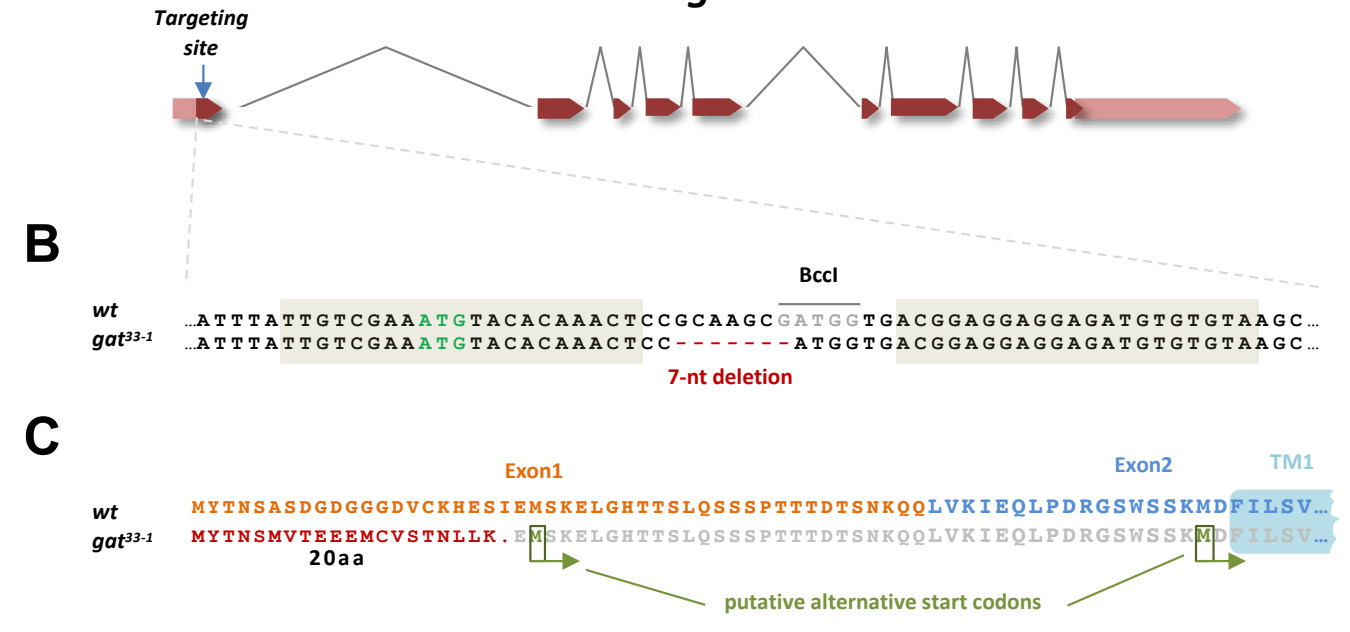
7-nt deletion

F

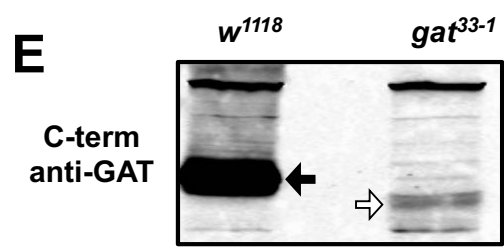

D
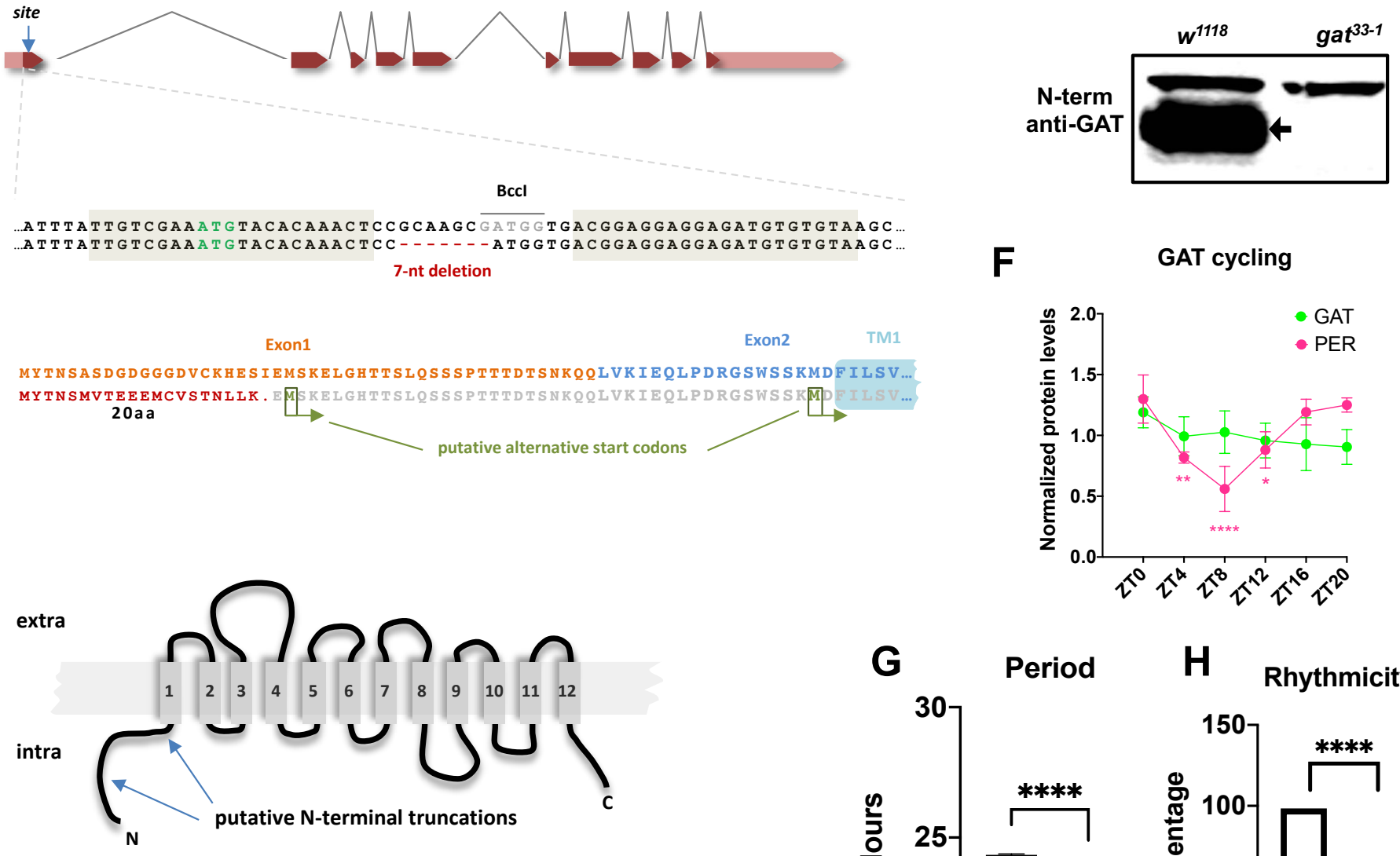

GAT cycling
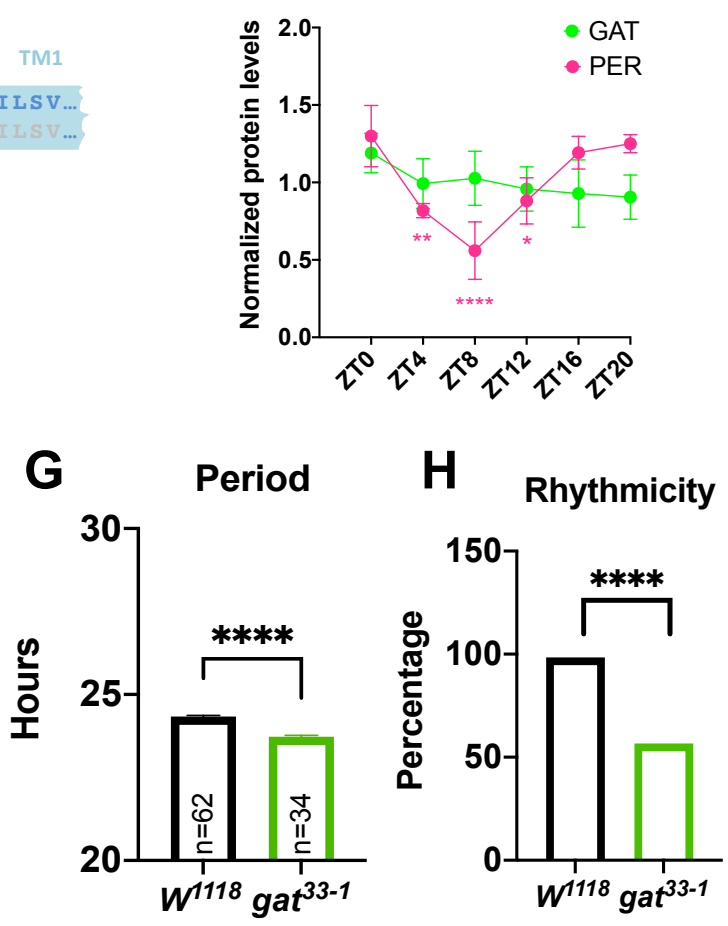
Figure 2.
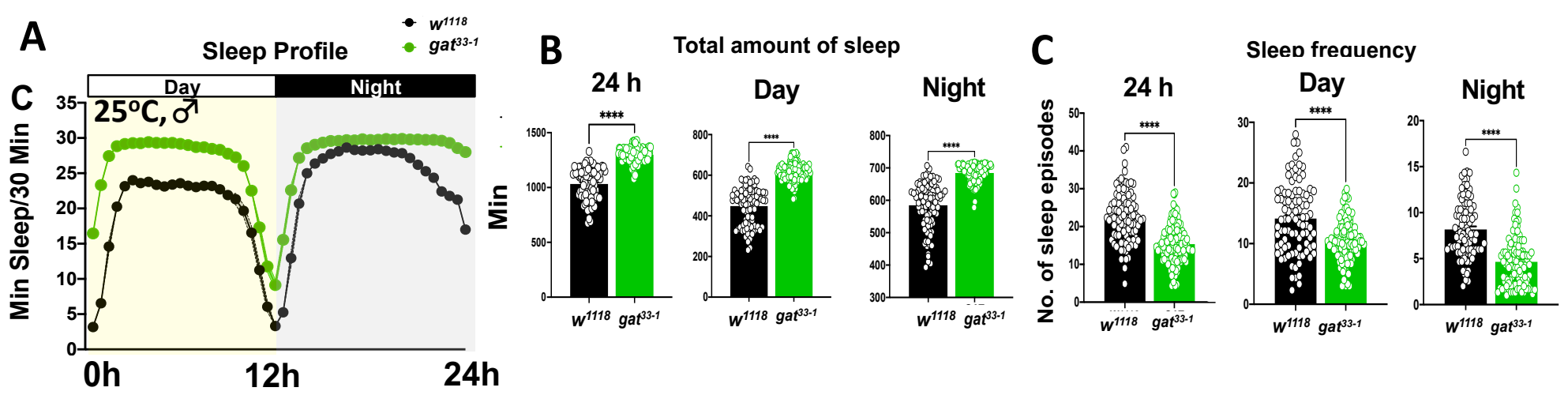

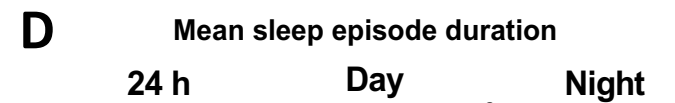

E

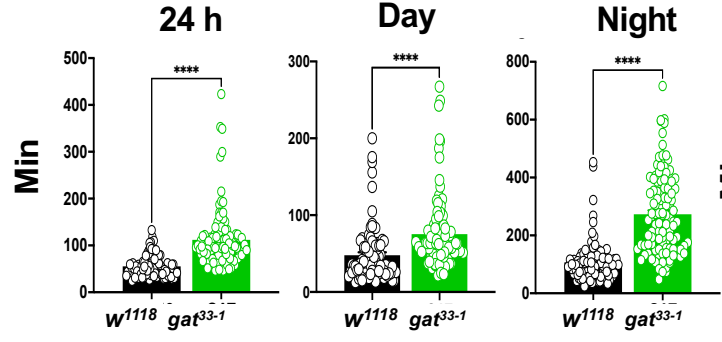

Mean wake episode duration

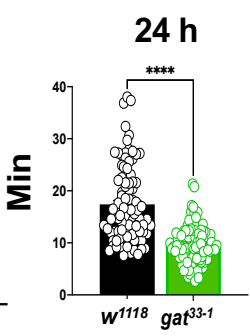

Day
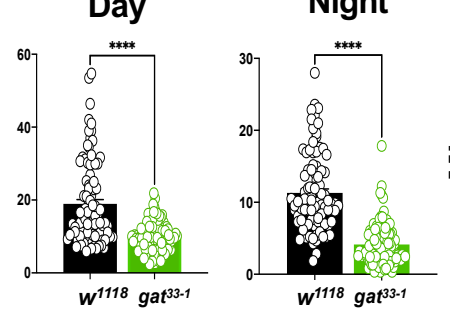

$\mathbf{F}$

Sleep latency

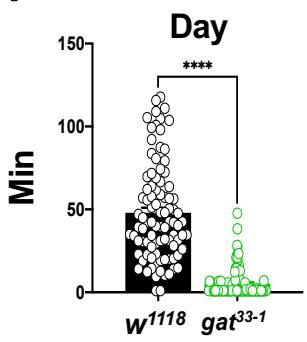

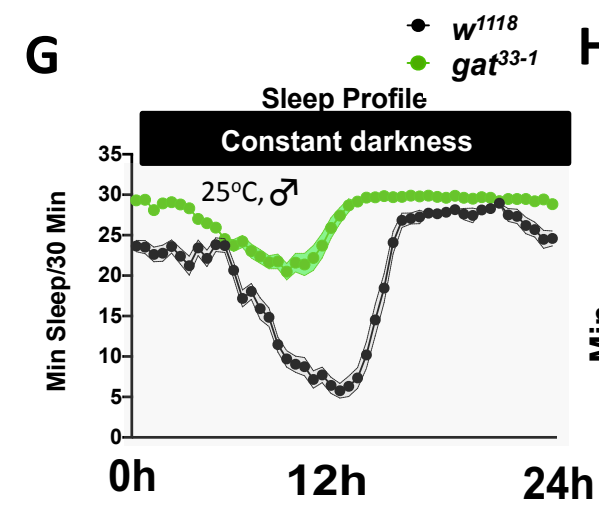

H

Total amount of sleep

$24 \mathrm{~h}$

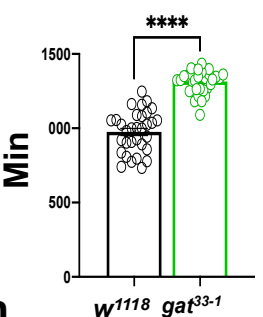

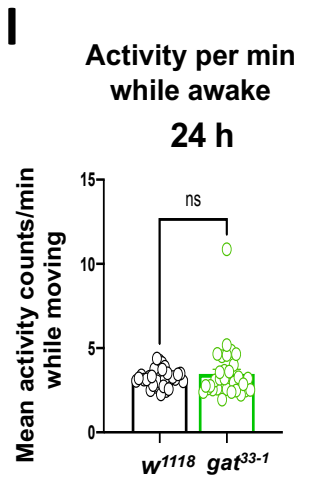

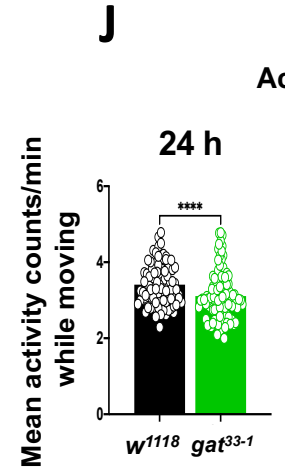

Activity per min while awake

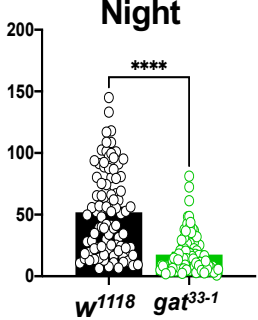

Day

Night
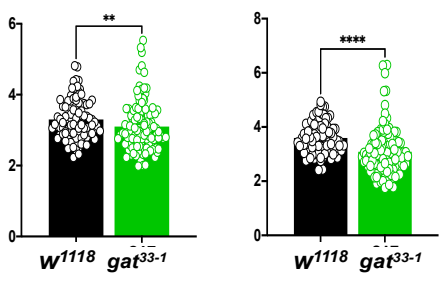
Figure 3.
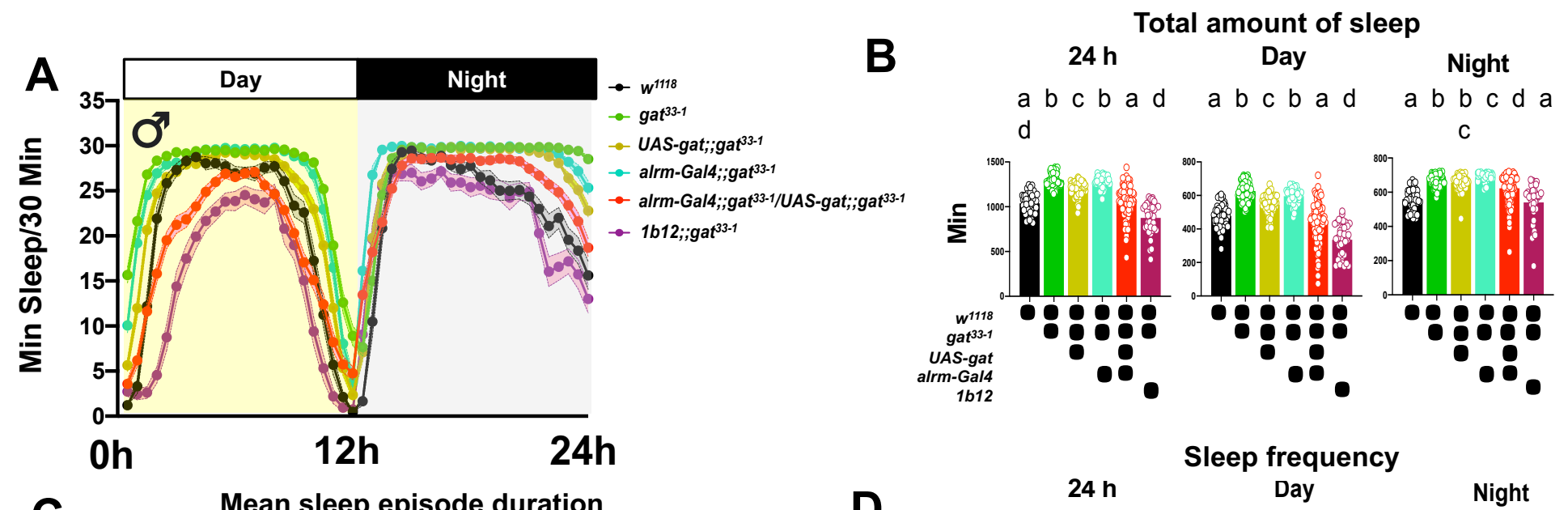

C

Mean sleep episode duration

D Night

$a b$ a b a c
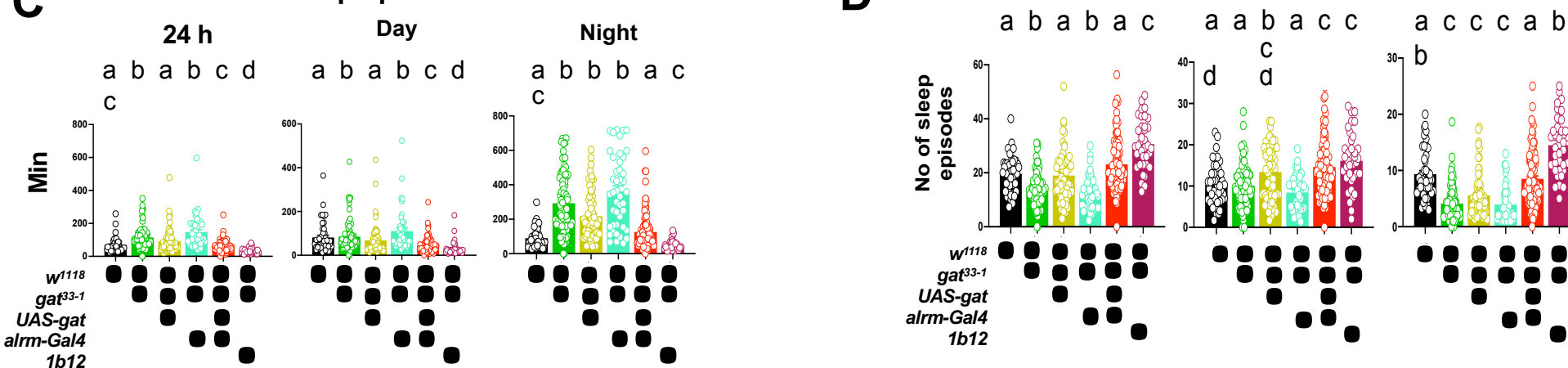

E

Sleep latency

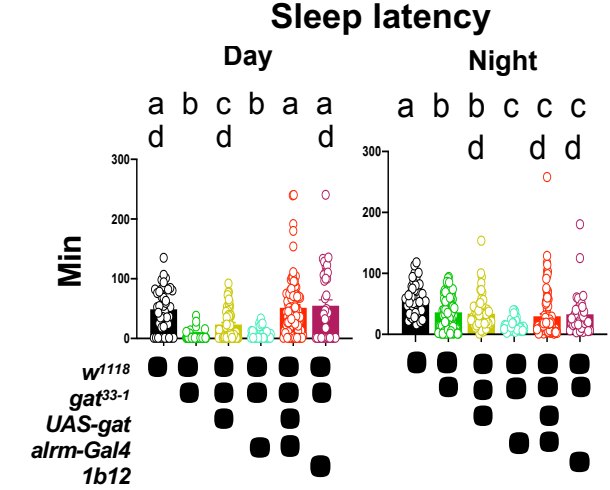

$F$

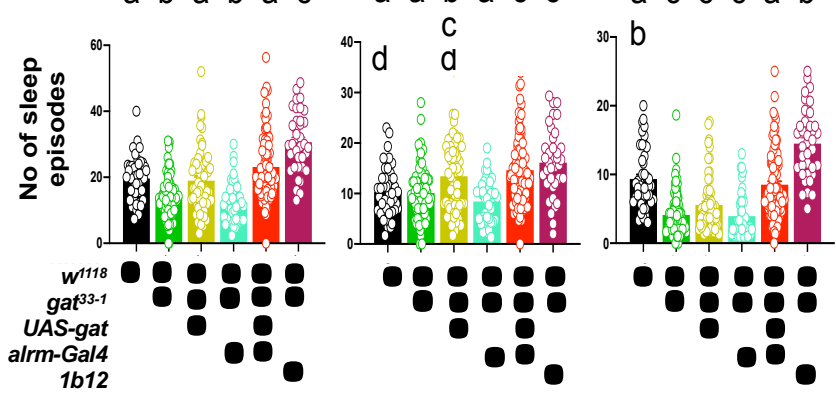

Activity per min while awake

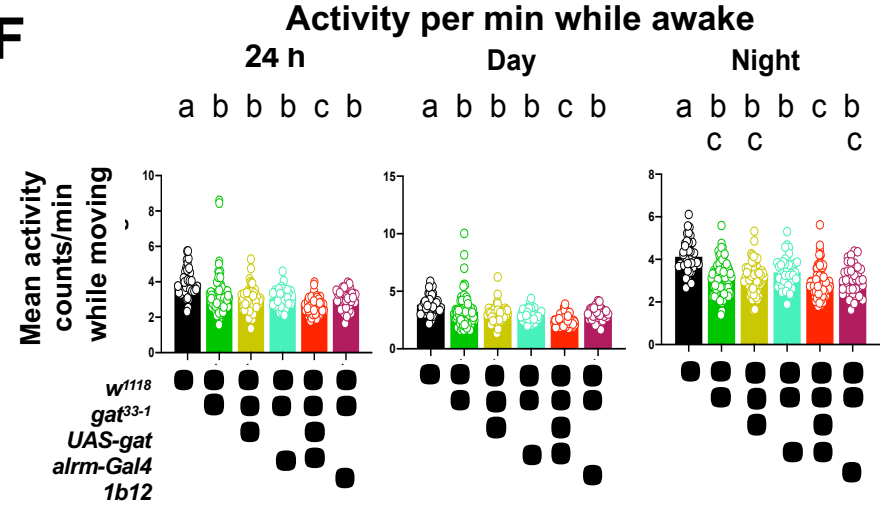




\section{Figure 4.}

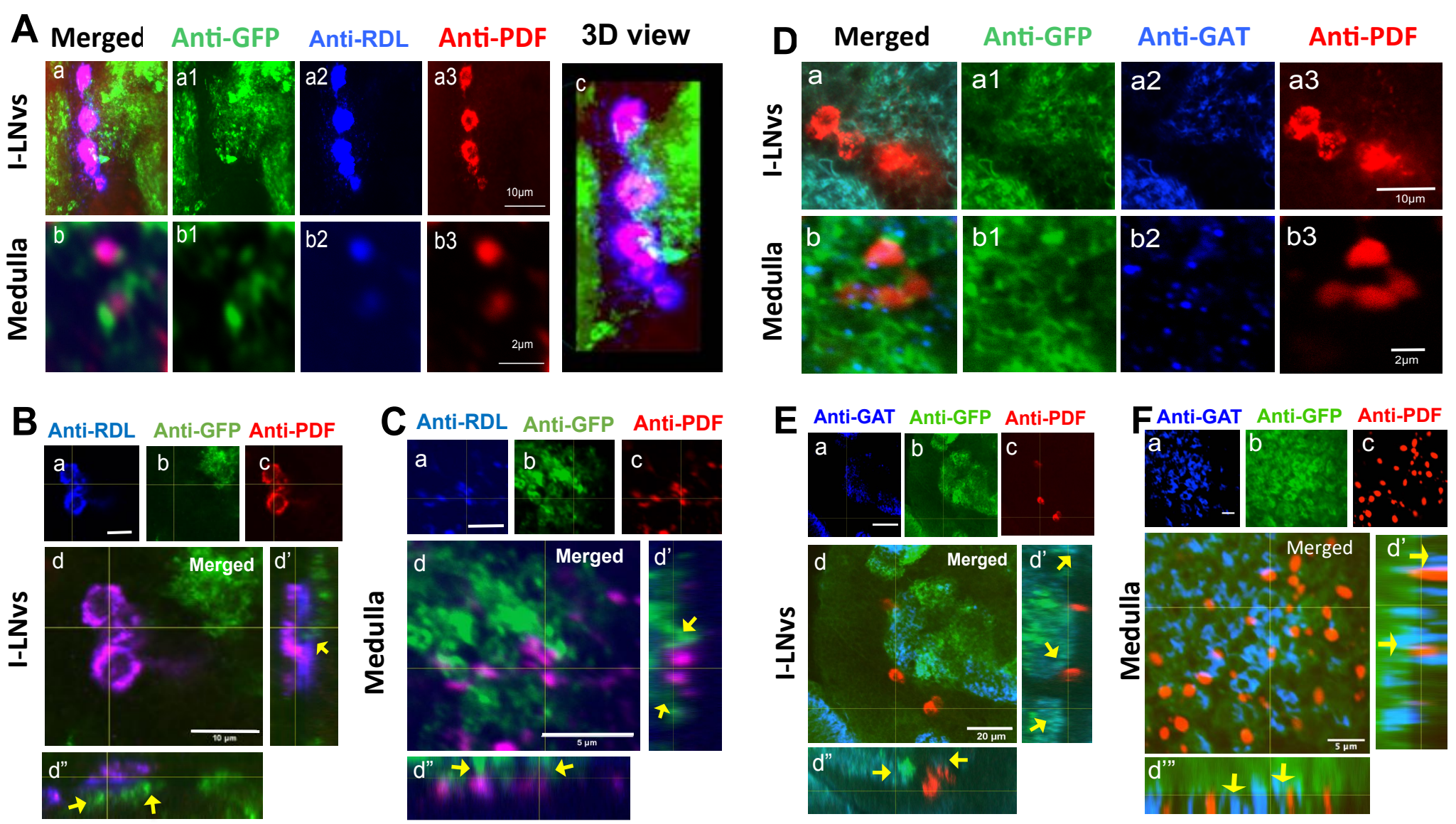


Figure 5.

A

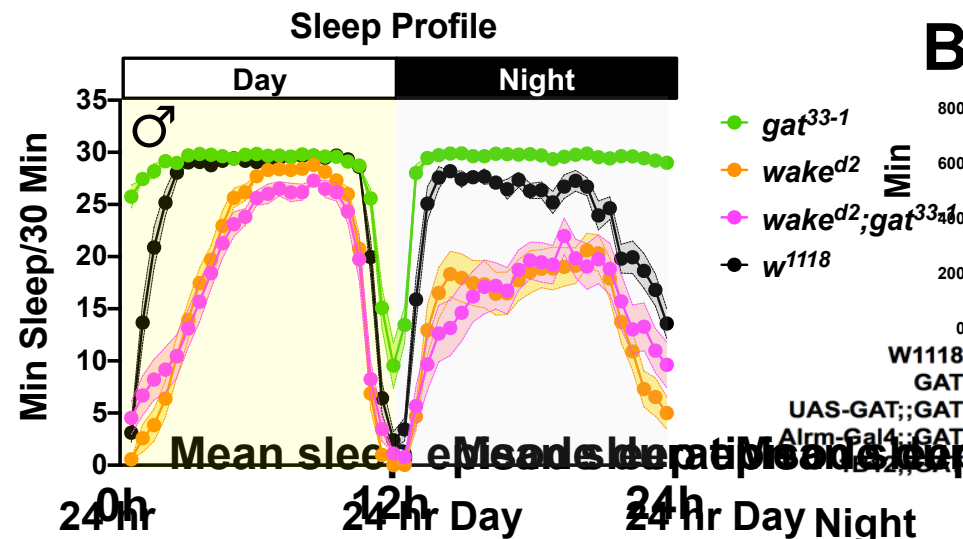

C

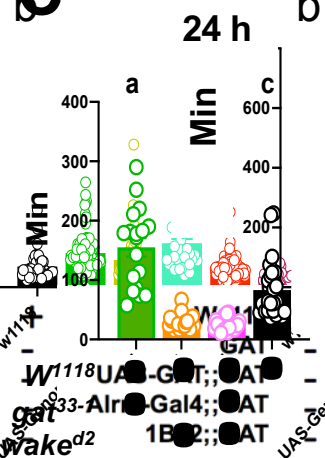

E

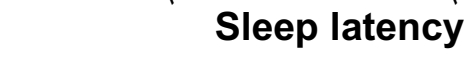

Day
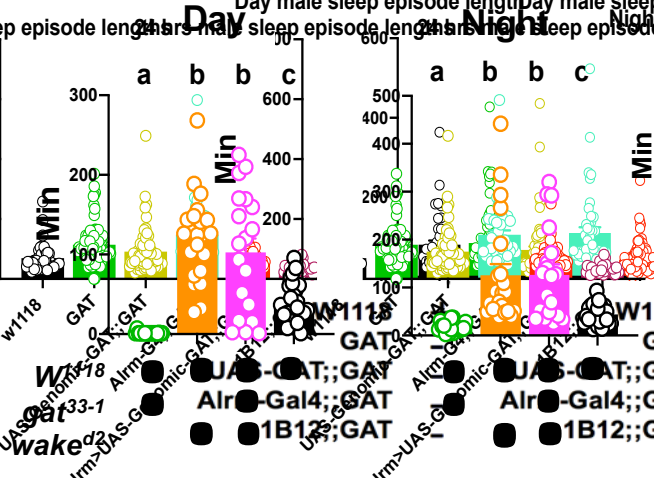

Night

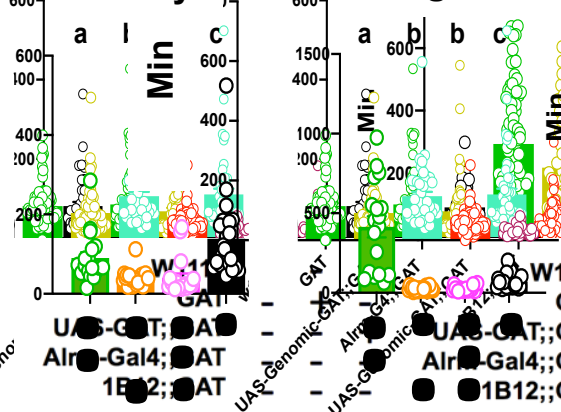

D

B

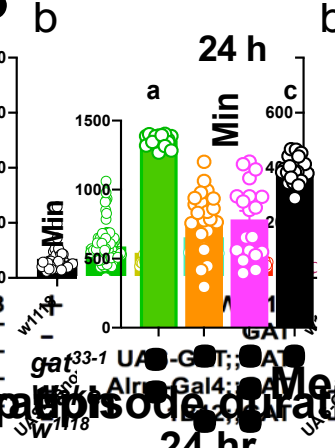

D

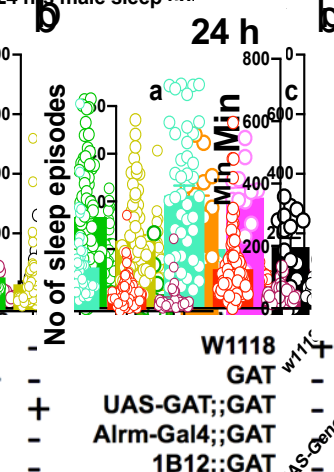

Sleep frequency

Day

Night
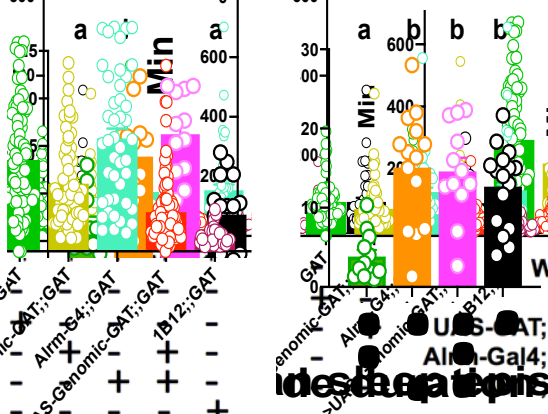

$\mathbf{F}$

Activity per min while awake
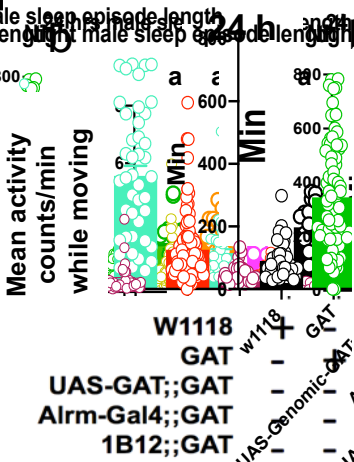

Day

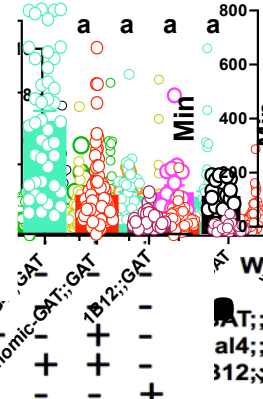

Night

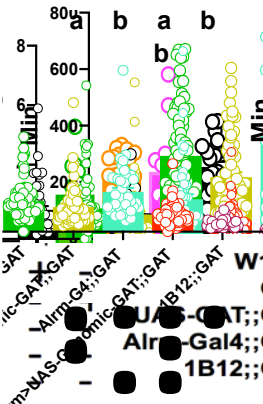




\section{Figure 6.}

A
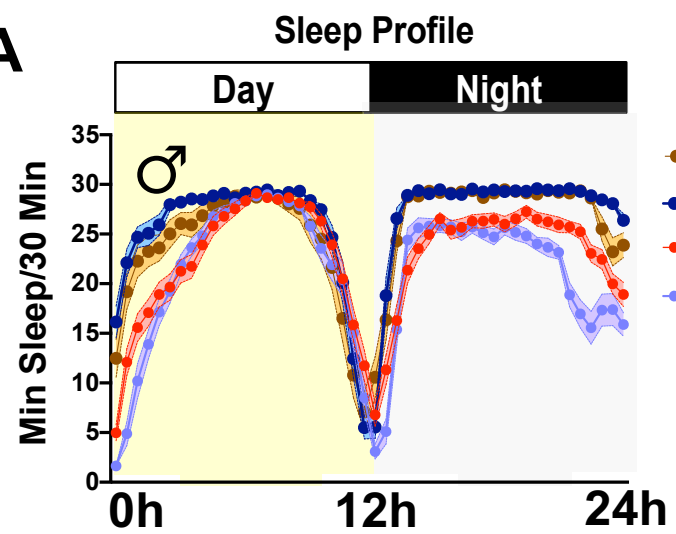

C

Mean sleep episode duration $24 \mathrm{~h}$

Day

Night

a $a$ b b
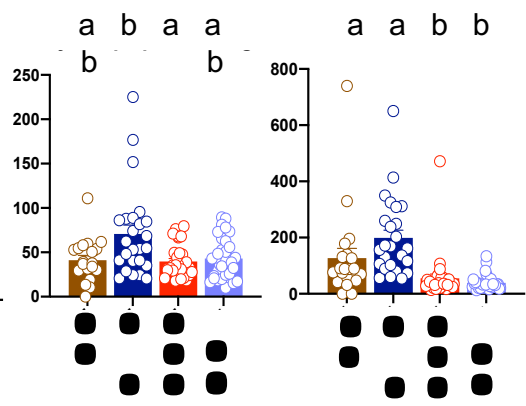

E

Sleep latency

$$
\text { Day Night }
$$

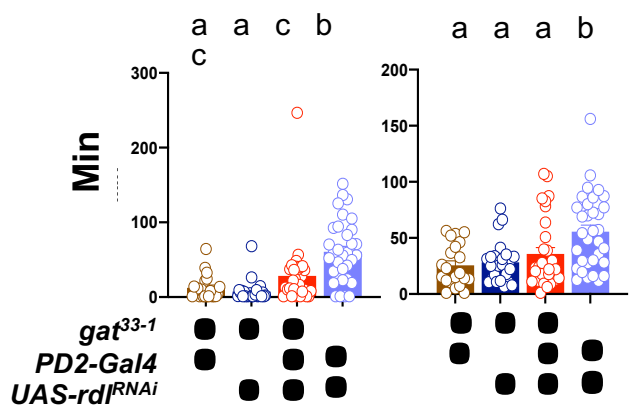

- PD2-Gal4;;gat $33-1$

- $r d l^{R N A i} ; ; g a t^{33-1}$

- PD2-Gal4;;gat ${ }^{33-1} /$ rdl $^{\text {RNAi } ; ; g a t^{33-1}}$

PD2-Gal4/rdI RNAi

D

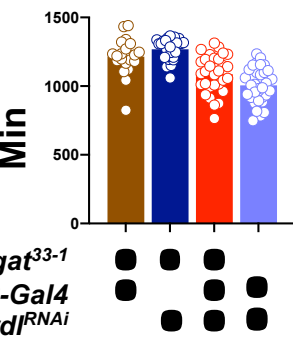
UAS-rdI ${ }^{R N A i}$ $24 \mathrm{~h}$

Sleep frequency

$a$ a b b

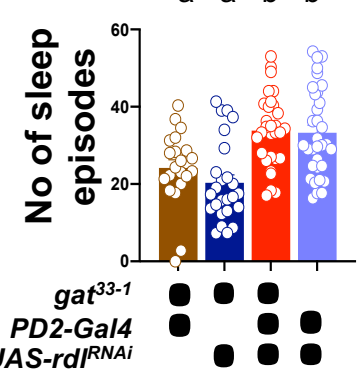

a a a a
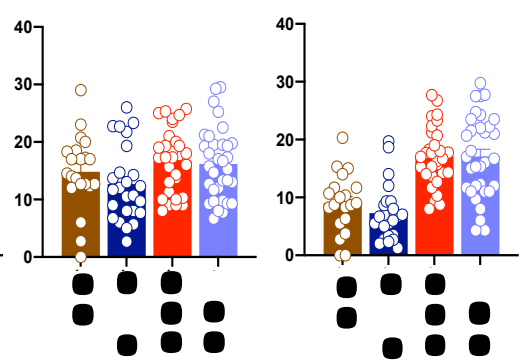

Activity per min while awake $24 \mathrm{~h}$

Day

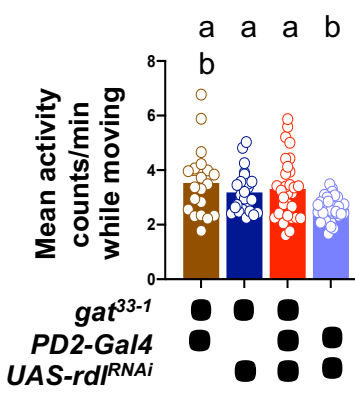

a $\quad a \quad a \quad b$

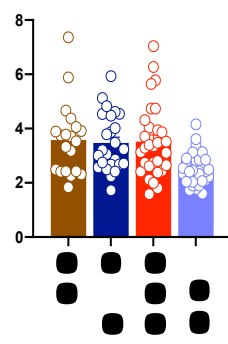

Night a $a$ b b

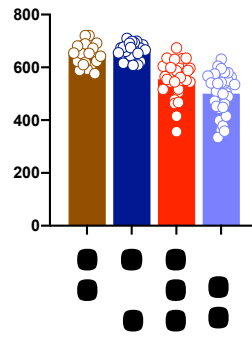

Night

Night a $a$ b b

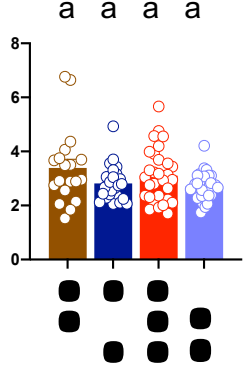


Figure 7.

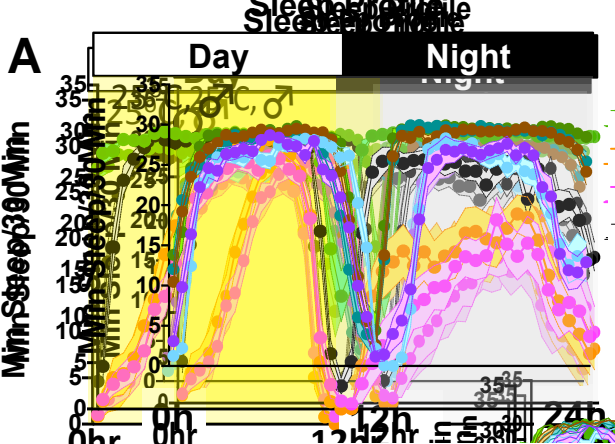

C

Mean sleep episode duration
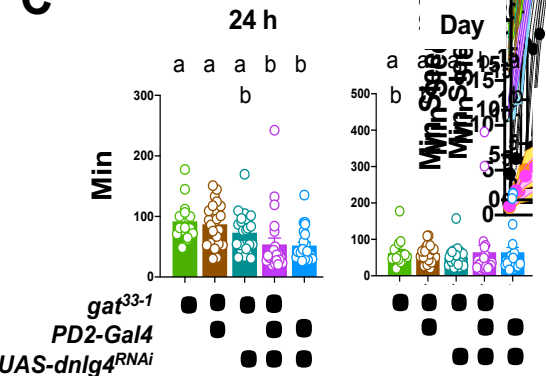

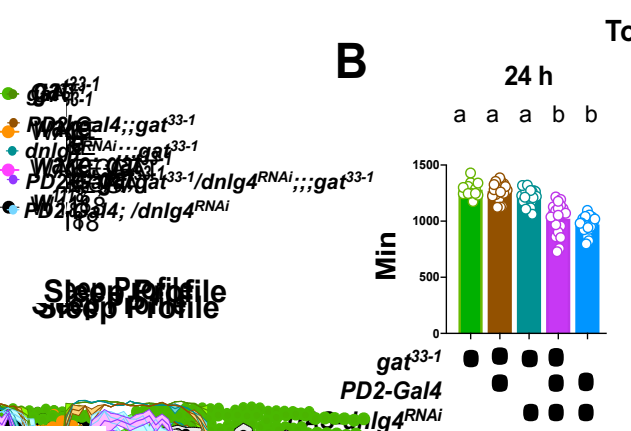

D

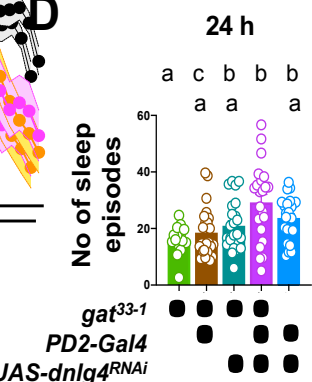

Total amount of sleep
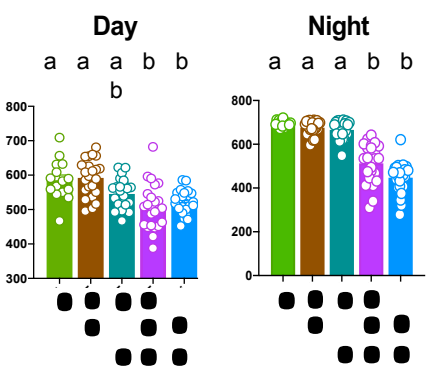

Sleep frequency

Day

Night

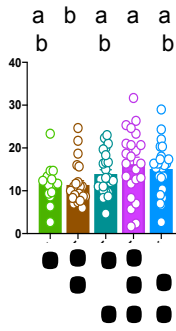

a a $a$ b a

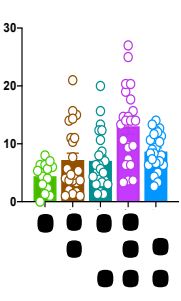

E

Maximum sleep episode duration

$24 \mathrm{~h}$

Day

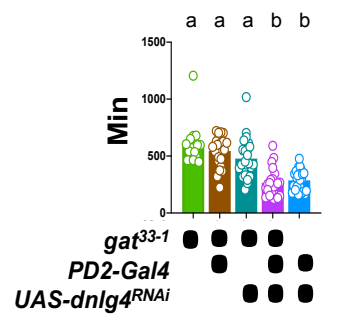

Night

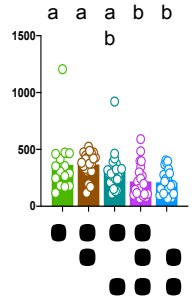

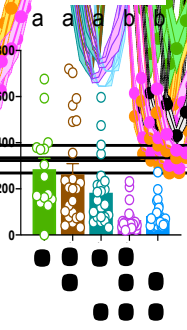

$\mathbf{F}$

Sleep latency
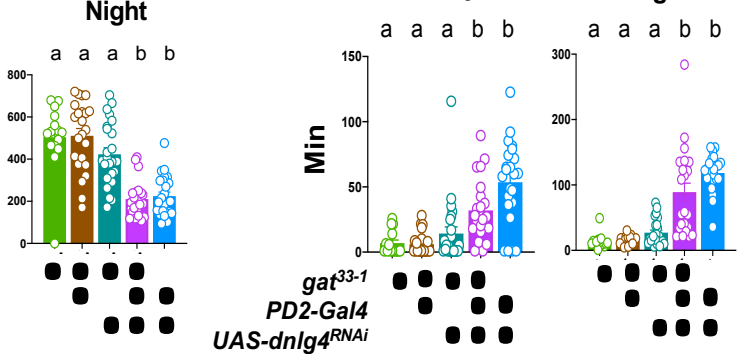

G

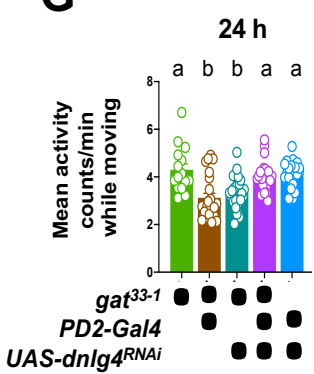

Activity per min while awake

Day
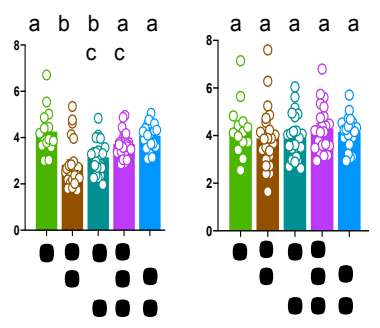

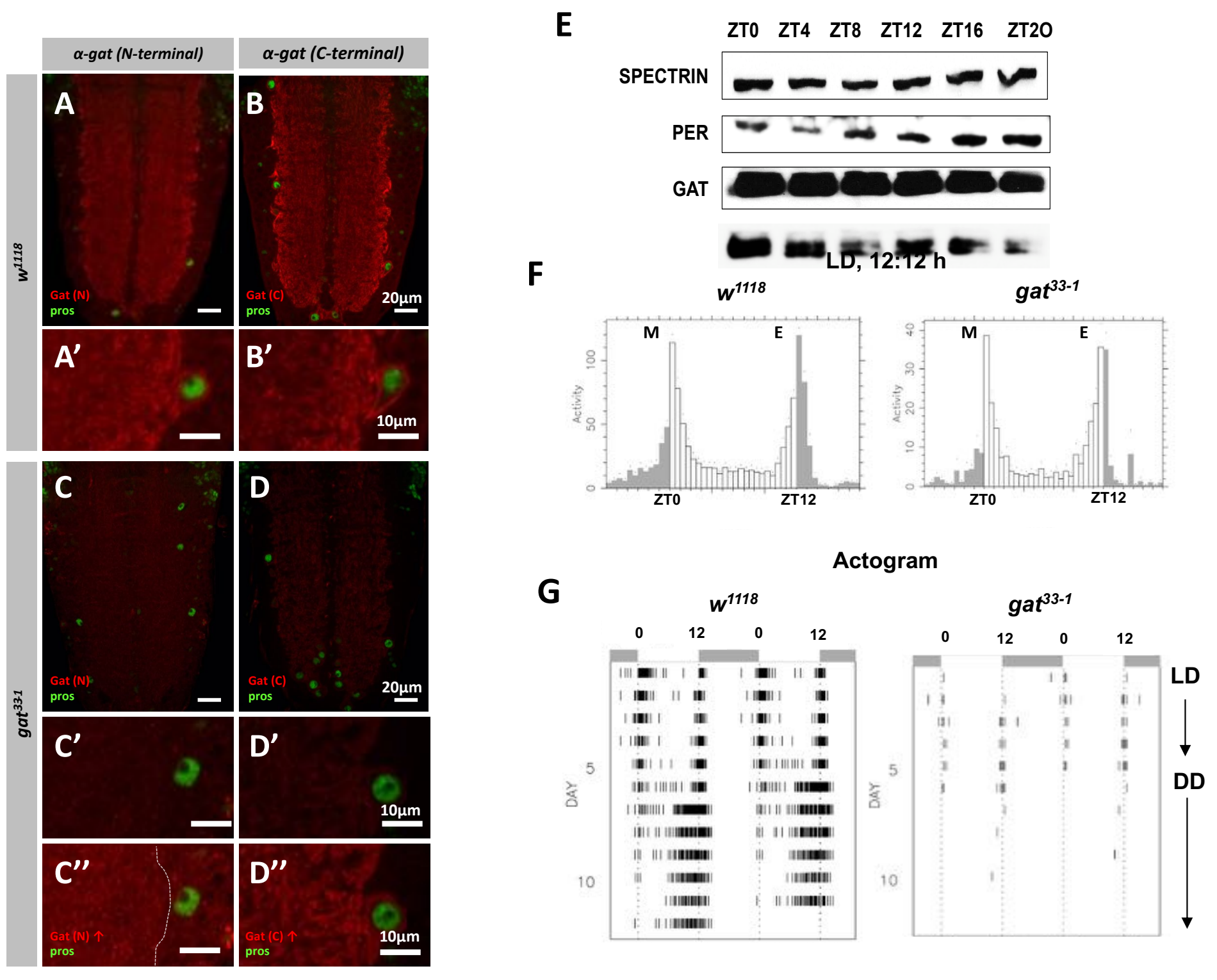


\section{Figure S1. Circadian characterization of gat $^{33-1}$ mutants and GAT expression:}

(A-D) Confocal analysis of L3 larval central nervous systems (VNCs). VNCs were labeled with antiPROS to identify astrocyte nuclei (A-D, green) and either anti-GAT N-terminus (A and C, red) or anti-GAT C-terminus (B and D, red) showing astrocyte membranes. (A, B, C, D) show overviews of the posterior region of the VNC, and (A'-D' and A"-D") show detailed views focusing on astrocytic cell bodies at the neuropil-cortex boundary. gat $^{33-1}$ mutants show a strong reduction in anti-GAT signal with both $\mathrm{N}$ - and C-terminus detecting anti-GAT antibodies (C and D, C' and D') compared to wild type controls (A and B, A' and B'). Despite the reduction of GAT protein levels in gat $^{33-1}$ hypomorphic mutants seen with the C-terminal anti-GAT antibody, enhancing the contrast reveals a relatively normal distribution of GAT protein within astrocytes seemingly labeling the cell membrane of the cell body and fine processes infiltrating the neuropil similar to wild type controls (B') while the anti-GAT N-terminal antibody shows a diffuse and unspecific signal in gat $^{33-1}$ mutants (C') compared to wild type controls (A'). (E) Western blot for GAT, PER, and Spectrin expression at different time points of the day. (F) Eduction plots of $g a t^{33-1}$ and control $w^{1118}$ male flies' showing the average activity of 16 flies/genotypes under a 12/12 h LD cycle. The morning (M) and evening (E) anticipatory behavior driven by circadian clock are intact in the gat mutant flies $(n=16)$. (G) Doubleplotted actograms for gat $^{33-1}$ and control $\mathrm{w}^{1118}$ male flies representing their circadian locomotor behavior. Flies were entrained to a $12 / 12 \mathrm{~h} \mathrm{LD}$ cycle and then released in constant darkness (DD) for 6 days $(n=16)$. 

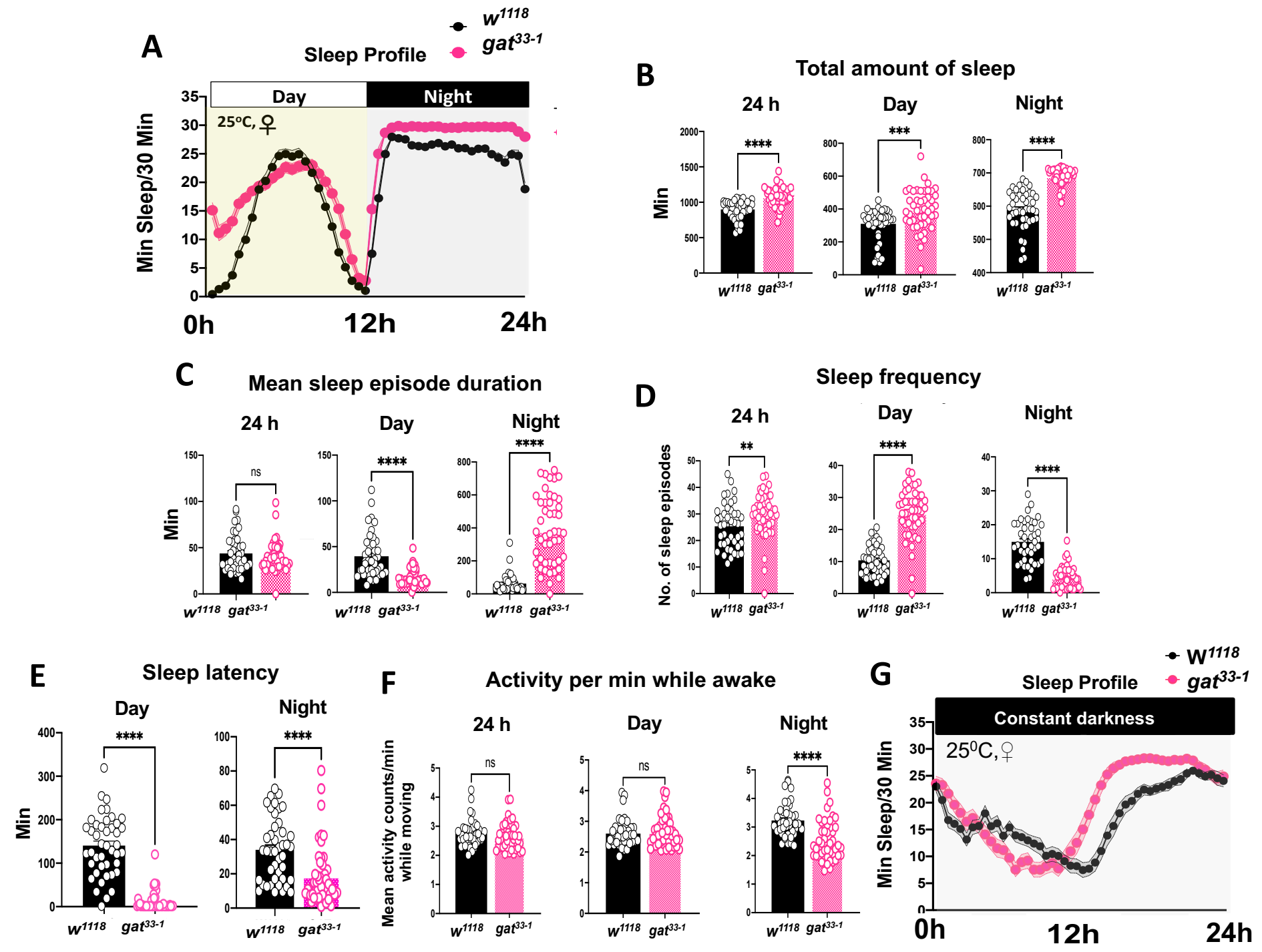

Sleep frequency

Figure S2. GABA transporter (GAT) is required for normal sleep-wake behavior

(A) Sleep profile of gat $^{33-1}$ (pink, $\mathrm{n}=48$ ), and control $\mathrm{w}^{1118}$ (black, $\mathrm{n}=43$ ) female flies in 12/12 h LD cycle showing enhanced sleep. (B-D) Sleep architecture during 24-hours (h) period, day, and night. Total sleep amount (min) (B), mean sleep episode duration ( $\mathrm{min}$ ) (C), and number of sleep episodes (beam breaks) (D). (E) Latency to sleep (min) during day, and nighttime. (F) Mean activity per min while awake (moving). (G) Sleep profile of gat $^{33-1}$ (pink, $n=32$ ), and control w ${ }^{1118}$ (black, $n=27$ ) female flies in constant darkness (DD). Error bar represents SEM. Statistical test as in figure 2. 


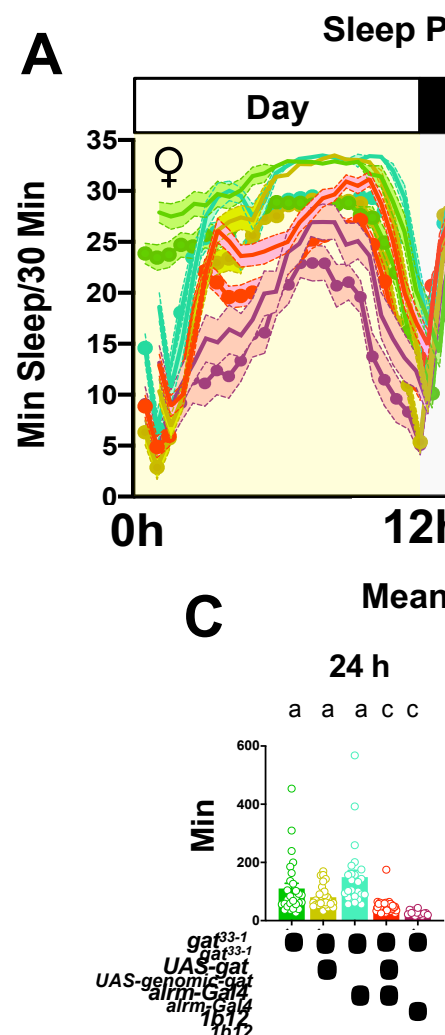

E $\begin{aligned} & \text { Sleep latency } \\ & \text { Day Night }\end{aligned}$

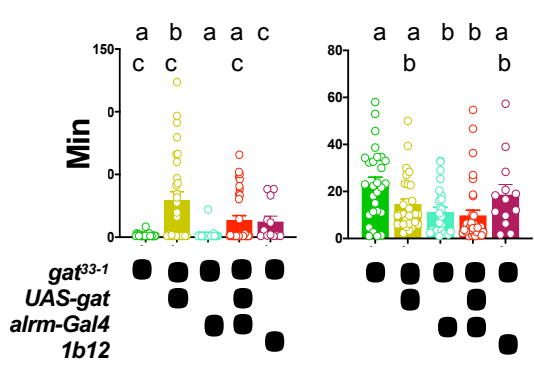

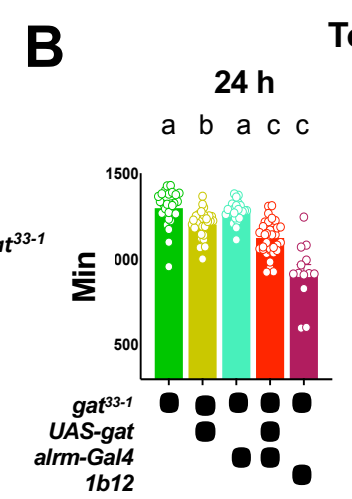

D

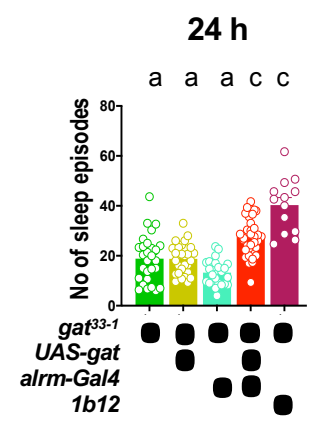

Sleep frequency

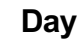

Night
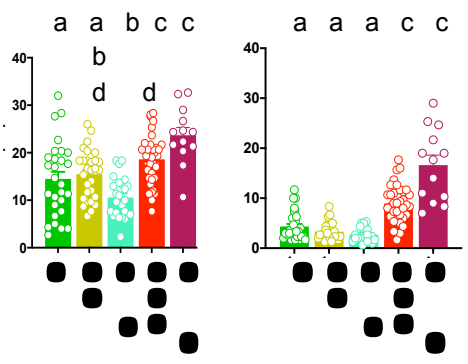

Night

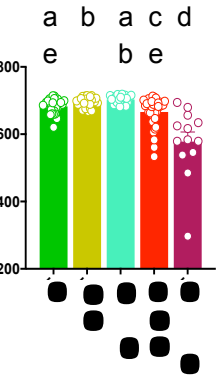

Activity per min while awake

$24 \mathrm{~h}$

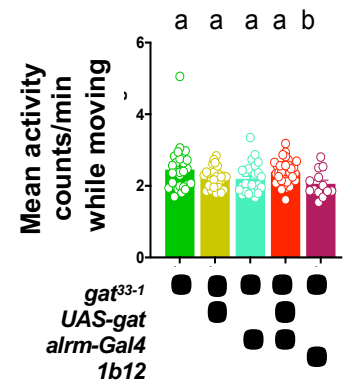

Day

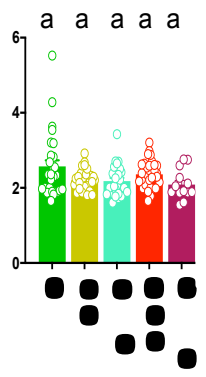

F

Night

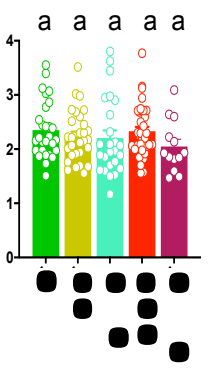

Figure S3. gat $^{33-1}$ sleep defects are rescued by expressing gat in astrocytes

(A) Sleep profile of female flies with genotype gat $^{33-1}$ (green, $\mathrm{n}=28$ ), UAS-gat;; gat $^{33-1}$ (olive, $\mathrm{n}=29$ ), alrm-Gal4;; gat $^{33-1}$ (sea-green, $\mathrm{n}=25$ ), alrm-Gal4;; gat ${ }^{33-1} / U A S-$ gat $;$; gat $^{33-1}$ (red, n=35), and 1b12;; gat ${ }^{33-1}$ (crimson, $\mathrm{n}=13$ ) in 12/12 h LD cycle. The results show that GAT functions in astrocytes. (B-D) Quantification of total amounts (min) of sleep (B), mean sleep episode's durations ( $\mathrm{min})(\mathrm{C})$, and number of sleep episodes (D) over 24-hours (h), during the day, and night. (E) Latency to sleep (min) during day and nighttime. (F) Mean activity per min while moving during 24-hour (h) period, day, and night. Error bar represents SEM. Statistics as in Figure 3. 
A
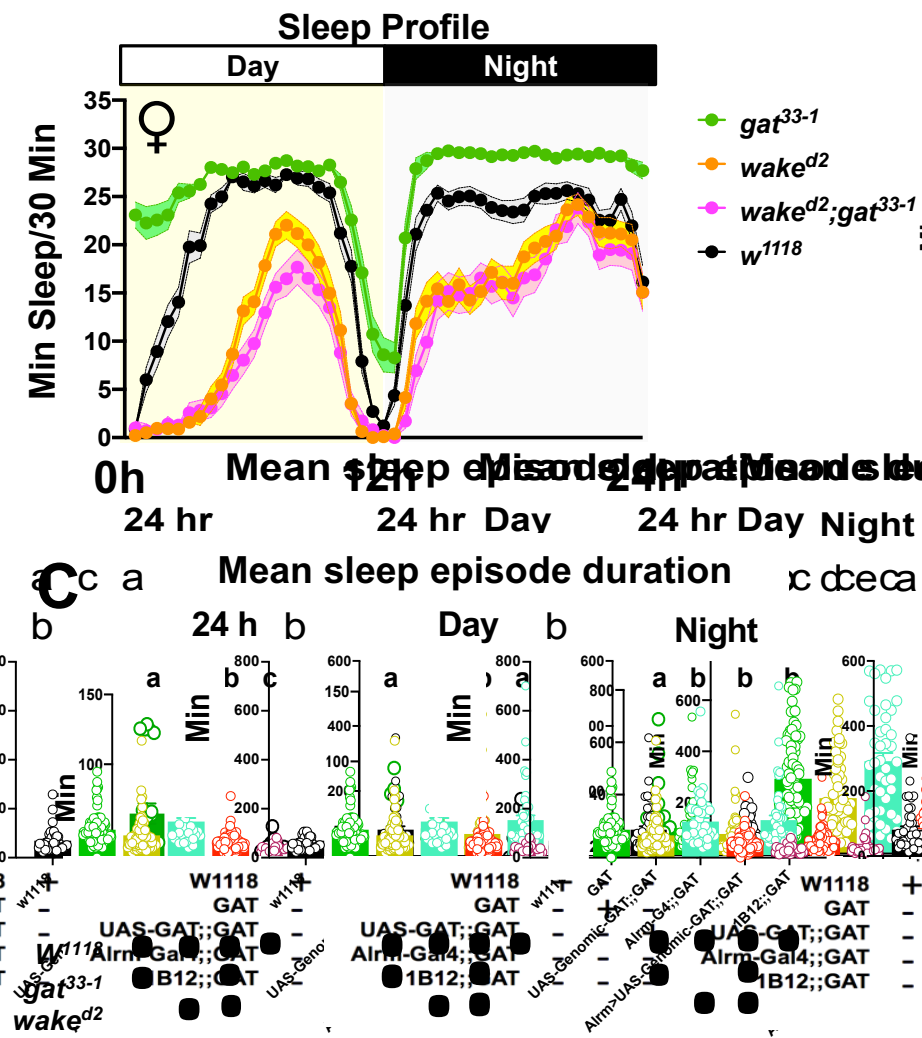

E

$\begin{array}{llll}\text { Day } & \text { Night } \\ \text { b } & \text { b } & \text { a } & \text { a }\end{array}$
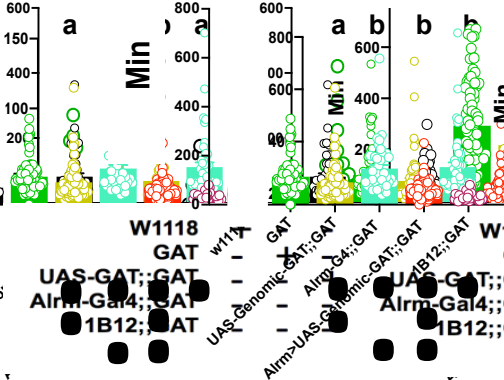

Sleep latency
B
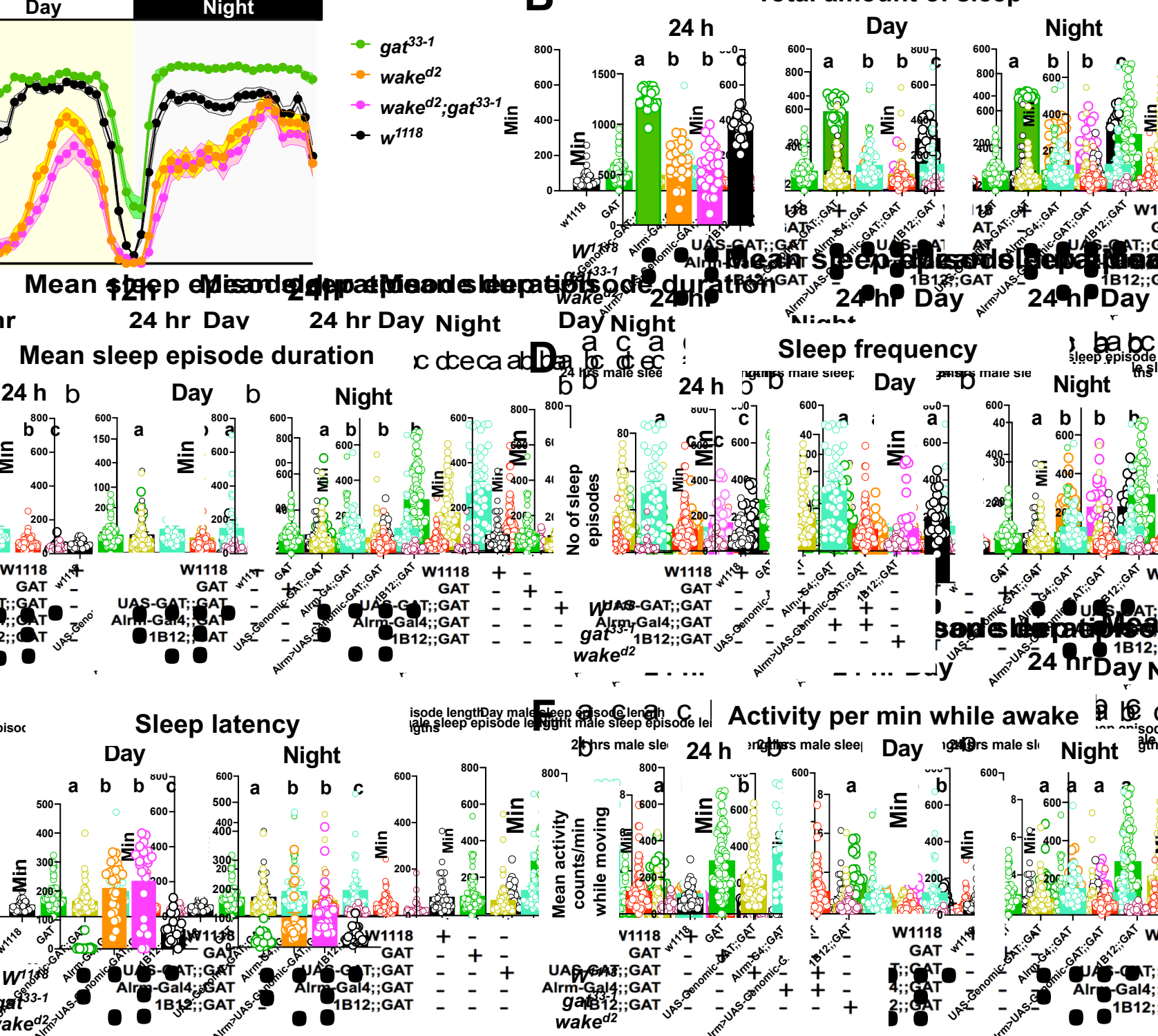

Night

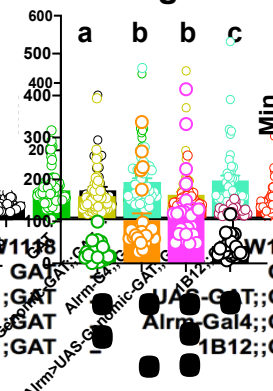

D

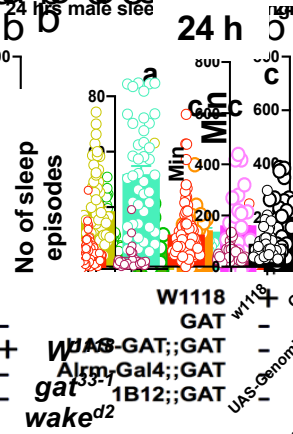

\section{Sleep frequency}

Day

Night
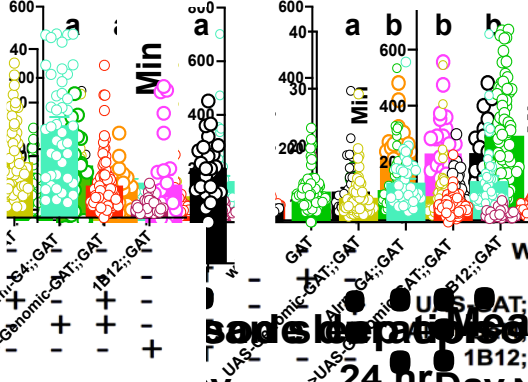

F

Activity per min while awake

$24 \mathrm{~h}$

Day

Night
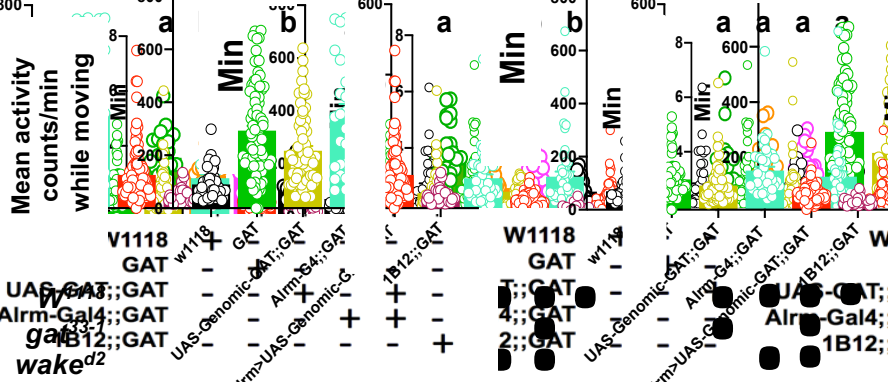

Figure S4. A wide wake (wake) mutation suppresses gat ${ }^{33-1}$ sleep phenotypes

(A) Sleep profile of female flies with genotype gat $^{33-1}$ (green, $\mathrm{n}=23$ ), control $w^{1118}$ (black, $\mathrm{n}=24$ ), wideawake mutant wake $^{d 2}$ (orange, $\mathrm{n}=24$ ), and double mutant with wake $^{d 2}$; gat $^{33-1}$ (pink, $\mathrm{n}=24$ ) in 12/12 h LD cycle. The results show that excess sleeping is suppressed in double mutant flies (B) Quantification of total sleep amounts (min) over 24 hours (h), day, and night. (C) Quantification of mean sleepepisode's durations lengths (min). (D) Number of sleep episodes (beam breaks) indicates fragmented sleep in wake $^{d 2}$ and double mutant flies. (E) Higher latency to sleep (min) shows difficulty in initiation of sleep in $w a k e^{d 2}$ and double mutant flies compared to controls. (F) Mean activity per min while moving during 24-hour (h) period, day, and night did not show any significant difference in wake ${ }^{d 2}$ flies, and double mutants from controls. Error bar represents SEM. Statistics as in Figure 3. 

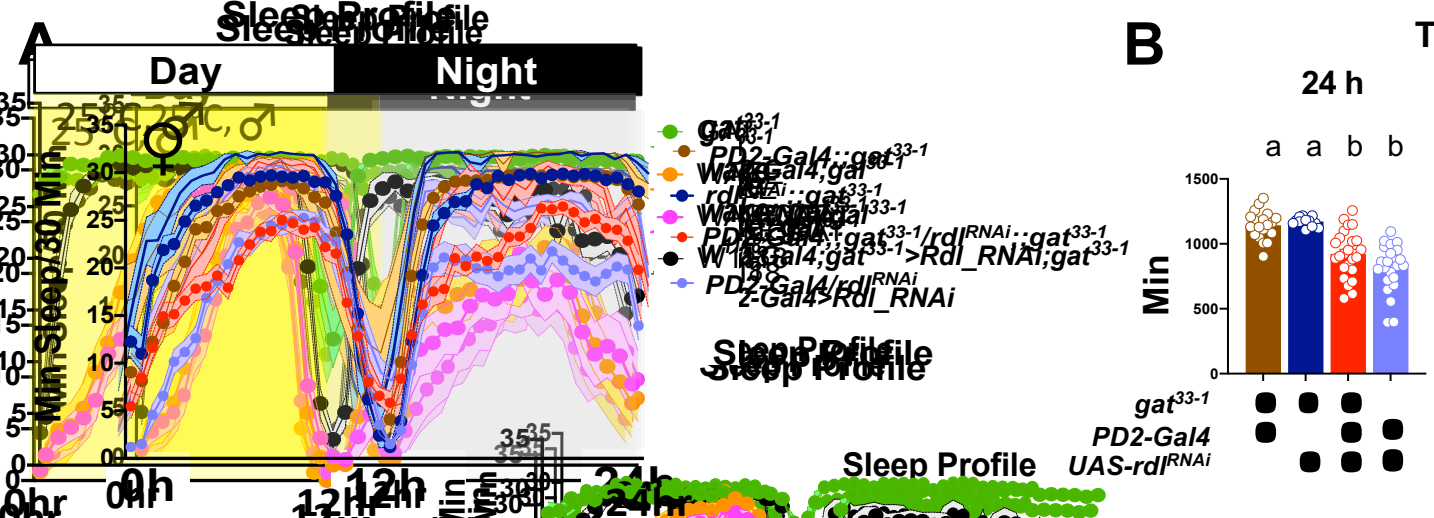

Total amount of sleep

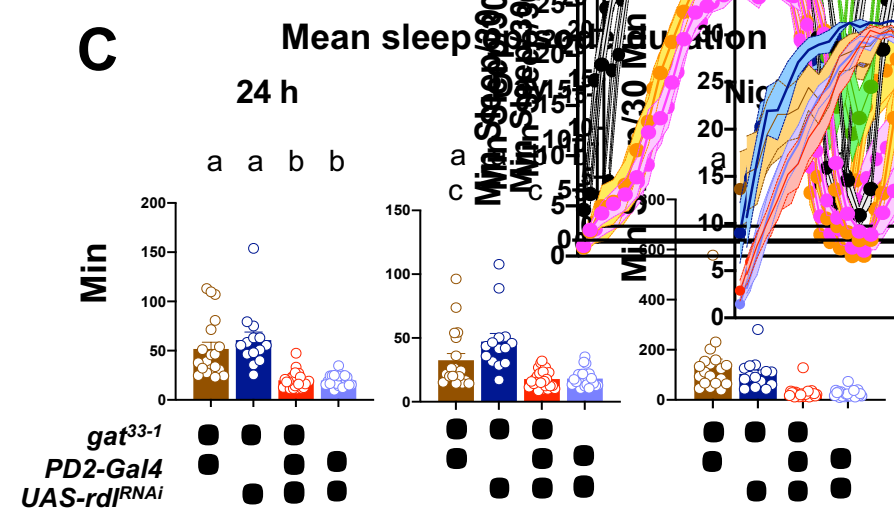

D
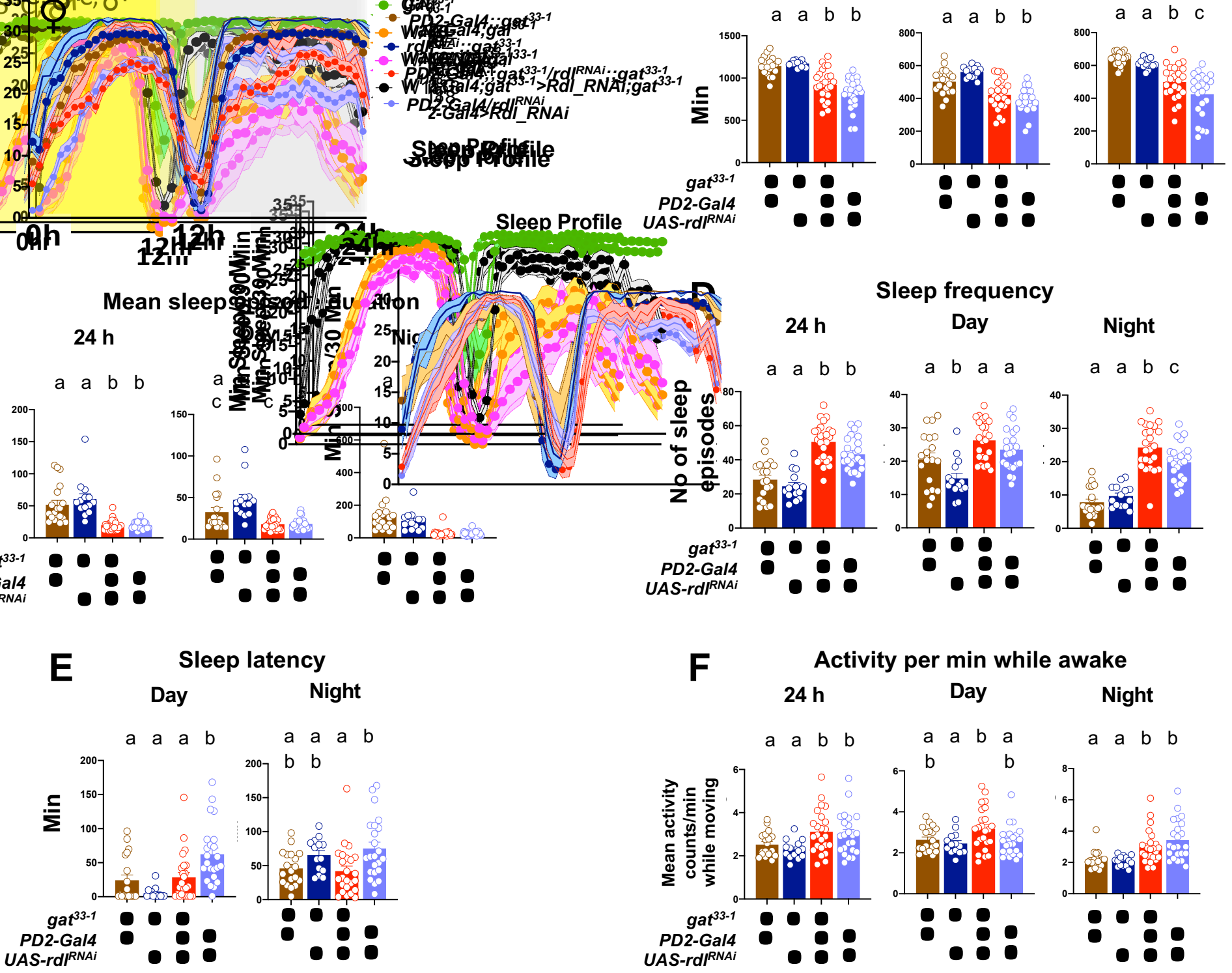

Sleep latency

Night

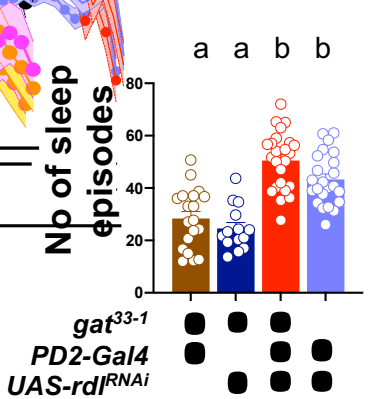

Sleep frequency

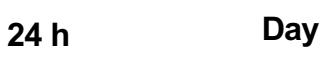

Night

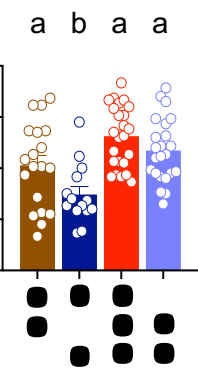

a

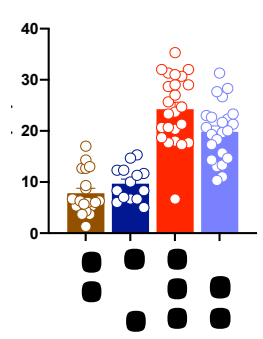

F Activity per min while awake

$24 \mathrm{~h}$

Day

Night
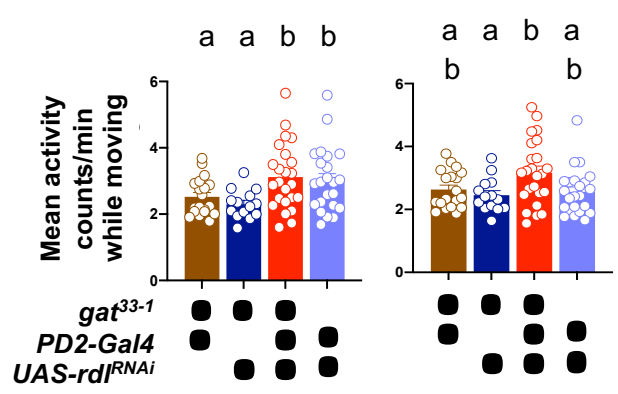

a $a$ b $b$

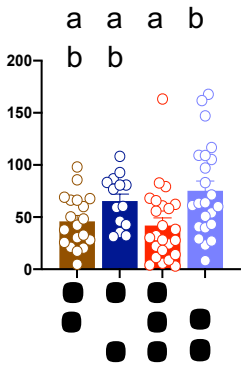

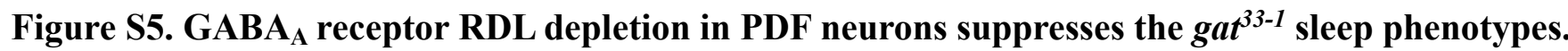

(A) Sleep profile of female flies with genotype PD2-Gal4;;gat ${ }^{33-1}$ (brown, $\mathrm{n}=19$ ), UAS-rdl ${ }^{R N A i} ;$;gat ${ }^{33-1}$ (navy blue, n=14), PD2-Gal4;;gat ${ }^{33-1}>U A S-r d l^{R N A i} ; ; g^{3} t^{33-1}$ (red, n=24), and PD2-Gal4>UAS-rdl ${ }^{R N A i}$ (lavender, $\mathrm{n}=23$ ) in 12/12 $\mathrm{h}$ LD cycle. The results show that excess sleeping is suppressed by $r d l$ RNAi knockdown in PDF neurons. (B) Quantification of total sleep amount (min) over 24 hours (h), during day, and during night. (C) Quantification of mean sleep episode's durations (min). (D) Number of sleep episodes indicate fragmented sleep in RDL knockdown flies with or without gat $^{33-1}$. (E) Latency to sleep (min) in RDL knockdown flies with and without gat $^{33-1}$. (F) Mean activity per min while moving during 24-hour (h) period, day, and night. Error bar represents SEM. Statistics as in Figure 3. 

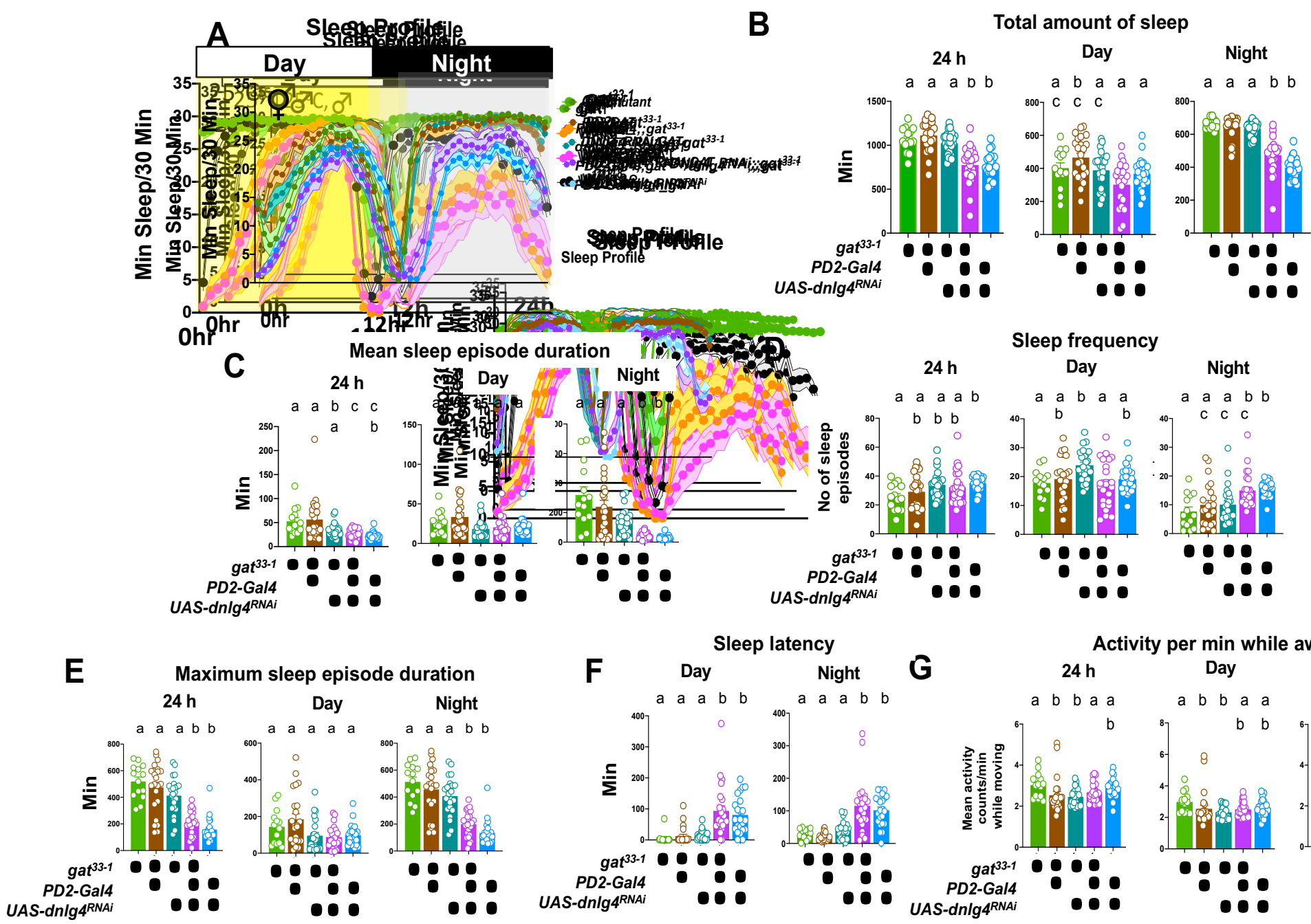

Sleep frequency
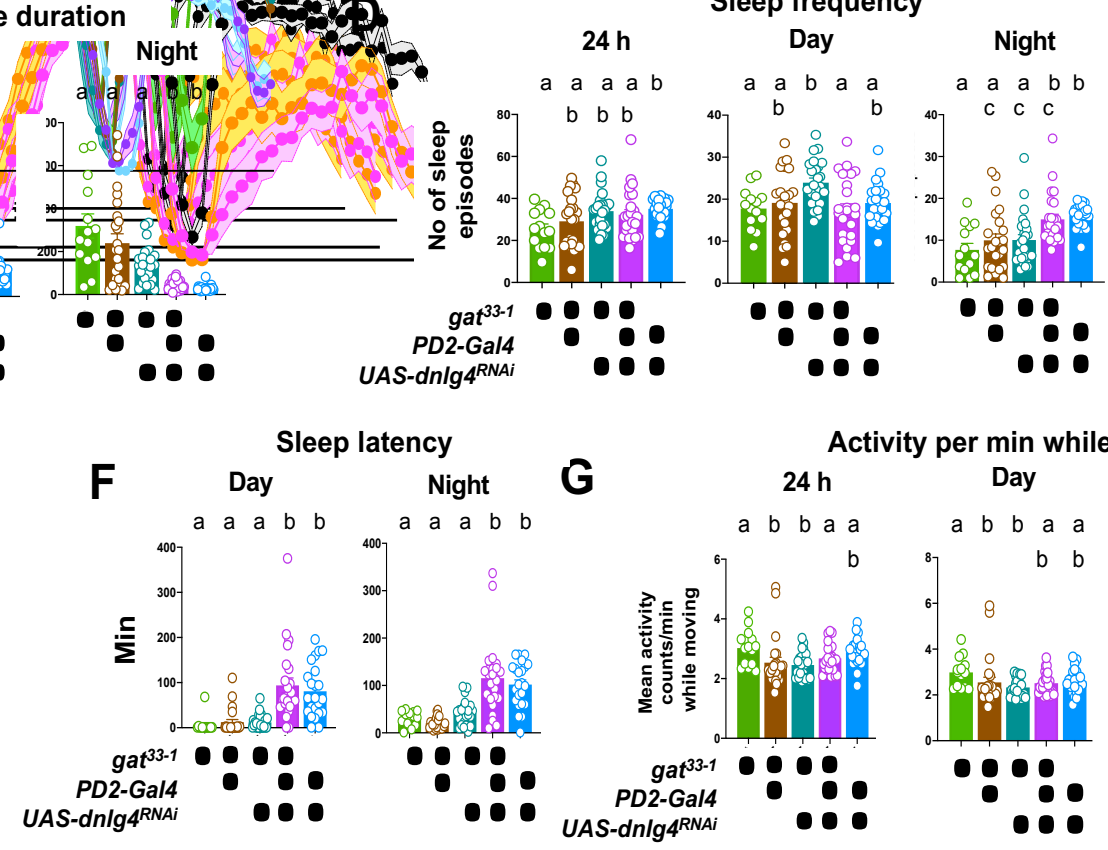

Activity per min while awake
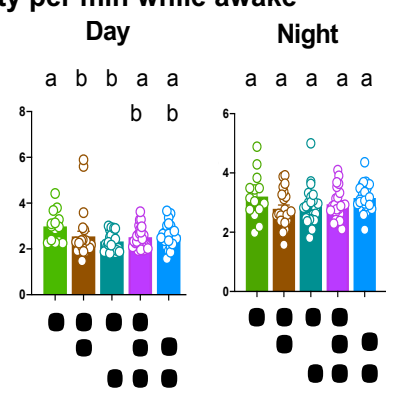

Figure S6. DNIg4 depletion in PDF neurons suppresses the gat $^{33-1}$ sleep phenotypes.

(A) Sleep profile of female flies with genotype gat $^{33-1}$ (green, $\mathrm{n}=14$ ), PD2-Gal4;; gat $^{33-1}$ (brown, n=23), $U A S$-dnlg4_RNAi;;;gat ${ }^{33-1}$ (teal, $\left.\mathrm{n}=24\right), P D 2-G a l 4 ; ; g^{3}{ }^{33-1}>d n l g 4 \_R N A i ; ; ; g a t^{33-1}$ (purple $\mathrm{n}=24$ ), and $P D 2-G a l 4>U A S-d n l g 4 \_R N A i$ (blue, $\mathrm{n}=23$ ) in 12/12 h LD cycle. The results show that excess sleeping is suppressed during nighttime by RNAi knockdown of neuroligin-4 ( $d n \lg 4)$ in PDF neurons (B) Quantification of total sleep amounts (min) over 24 hours (h), during day, and during night. Results show reduction in sleep during night in $d n \lg 4$ knockdown flies with and without gat $^{33-1}$. (C) Quantification of mean sleep-episode's durations (min). (D) Number of sleep episodes. (E) Maximum sleep episode's duration (min). (F) Higher latency to sleep (min) shows difficulty in initiation of sleep in $d n \lg 4$ knockdown flies with and without $g a t^{33-1}$. (G) Mean activity per min while moving during 24hour (h) period, day, and night. Statistics as in Figure 3. 


\section{Video-S1. Astrocytes are surrounding RDL, and PDF expressing clock neurons.}

Video demonstrating RDL (red color labeled with anti-RDL), and PDF (blue color labeled with anti-PDF) are present in clock neurons (s-LNvs and 1-LNvs). 1-LNvs send their projections in the medulla of the optic lobe. Astrocytes (alrm-Gal4>UAS-CD8-GFP, Green color, labeled with anti-GFP) are close to RDL and PDF positive neurons.

Video-S2. Astrocytes are surrounding RDL and PDF expressing l-LNvs terminals present in the optic lobe's medulla.

Video demonstrating that RDL (red), and PDF (blue) in 1-LNvs projection are very close to astrocytes (alrm-Gal4>UAS-CD8-GFP, Green color) in the medulla region.

\section{Video-S3. Magnified view of astrocytes expressing GAT in the adult fly brain}

Video showing GAT (blue) is present in astrocytes (green, alrm-Gal4>UAS-CD8GFP). PDF marks the s- and 1-LNv cell bodies in red.

\section{Video-S4. Astrocytes are expressing GAT in the medulla of the optic lobe.}

Video showing GAT (blue) in astrocytes is labeled with anti-GAT. Astrocytes are marked with anti-GFP (green color, alrm-Gal4>UAS-CD8GFP). PDF (red color) labels the I-LNvs neuronal projections innervating into medulla.

\section{Video-S5. Astrocytes expressing GAT in the adult fly brain}

Video showing GAT (blue) labeled with anti-GAT and present in astrocytes (green color, alrmGal4>UAS-CD8GFP) labeled with anti-GFP. The video captures the whole fly brain. 